\title{
Mesoscale Simulation of Tropical Cyclones in the South Pacific: Climatology and Interannual Variability
}

\author{
Nicolas C. Jourdain, ${ }^{*}$ Patrick Marchesiello, ${ }^{*},+$ Christophe E. MenKes, ${ }^{*}, \#$ Jérome Lefèvre,*

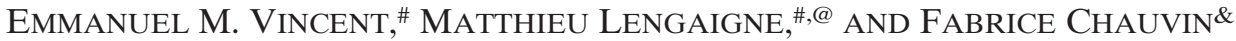 \\ * Institut de Recherche pour le Développement, Nouméa, New Caledonia \\ ${ }^{+}$Laboratoire d'Etudes en Géophysique et Océanographie Spatiale, Toulouse, France \\ \# Laboratoire d'Océanographie et du Climat: Expérimentation et Approches Numériques, Paris, France \\ ${ }^{@}$ National Institute of Oceanography, Goa, India \\ \& Centre National de Recherches Météorologiques, Toulouse, France
}

(Manuscript received 9 December 2009, in final form 4 August 2010)

\begin{abstract}
The Weather Research and Forecast model at $1 / 3^{\circ}$ resolution is used to simulate the statistics of tropical cyclone (TC) activity in the present climate of the South Pacific. In addition to the large-scale conditions, the model is shown to reproduce a wide range of mesoscale convective systems. Tropical cyclones grow from the most intense of these systems formed along the South Pacific convergence zone (SPCZ) and sometimes develop into hurricanes. The three-dimensional structure of simulated tropical cyclones is in excellent agreement with dropsondes and satellite observations. The mean seasonal and spatial distributions of TC genesis and occurrence are also in good agreement with the Joint Typhoon Warning Center (JTWC) data. It is noted, however, that the spatial pattern of TC activity is shifted to the northeast because of a similar bias in the environmental forcing. Over the whole genesis area, $8.2 \pm 3.5$ cyclones are produced seasonally in the model, compared with $6.6 \pm 3.0$ in the JTWC data. Part of the interannual variability is associated with El NiñoSouthern Oscillation (ENSO). ENSO-driven displacement of the SPCZ position produces a dipole pattern of correlation and results in a weaker correlation when the opposing correlations of the dipole are amalgamated over the entire South Pacific region. As a result, environmentally forced variability at the regional scale is

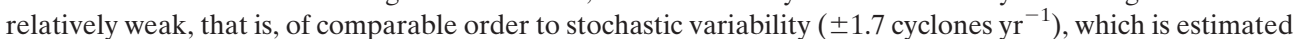
from a 10 -yr climatological simulation. Stochastic variability appears essentially related to mesoscale interactions, which also affect TC tracks and the resulting occurrence.
\end{abstract}

\section{Introduction}

Thirty years ago, Gray (1979) showed that the seasonal frequency of tropical cyclone (TC) genesis at any location is closely related to large-scale environmental parameters. Based on this study, genesis indices were developed for seasonal predictions (Watterson et al. 1995; Camargo et al. 2007a) and climate projections (Royer et al. 1998). However, as noted by Ooyama (1982), the synoptic conditions do not directly determine the genesis process, but only affect the probability of its happening. Suitable environmental conditions are generally present in monsoonal genesis regions, such as the western South Pacific, but are not sufficient. Triggering mechanisms are also needed to

Corresponding author address: Nicolas Jourdain, IRD, Nouméa Center, BP A5, Nouméa CEDEX, New Caledonia.

E-mail: nicolas_jourdain@yahoo.fr overcome the obstacle of relatively low midtropospheric humidity. They appear in the form of externally forced convergence, such as wind surges in the trade winds or monsoon flow, or/and mesoscale interactions (Gray 1998; Simpson et al. 1997). Simpson et al. (1997) use the few available observations in the western South Pacific to suggest that interactions of mesoscale convective systems (MCSs) play a crucial role in TC genesis once favorable large-scale conditions have been established [large scales refer to structures larger than about $2000 \mathrm{~km}$, following the subdivision of Orlanski (1975)]. These mesoscale interactions are essentially stochastic in nature, although the degree of stochasticity may be reduced by environmental influences. In the Atlantic region, tropical cyclones often form from tropical waves moving off of Africa (Landsea 1993). In this case, TC genesis appears to rely less on mesoscale stochastic processes and more on forced background conditions, so that predictability seems higher in 
the Atlantic than in the Pacific (Vitart et al. 1997; Knutson et al. 2007; Zhao et al. 2009). Specifically, Zhao et al. (2009) found correlations greater than 0.8 between observed and simulated yearly hurricane counts in the North Atlantic, but only 0.3 in the South Pacific.

Many climatological studies do not consider mesoscale interactions because they rely on atmospheric general circulation models (AGCMs) of coarse horizontal resolution that simulate cyclone-like vortices, that is, vortices that have some characteristics of tropical cyclones but much lower intensities (e.g., Wu and Lau 1992; Bengtsson et al. 1996; Vitart et al. 1997; Sugi et al. 2002; Camargo et al. 2005; Yoshimura et al. 2006). Their relevance to actual TC activity remains difficult to assess (Gray 1998; Camargo et al. 2007b). More recent studies have shown that increasing AGCM resolution to around $50 \mathrm{~km}$ improves the representation of simulated cyclones (Oouchi et al. 2006; Chauvin et al. 2006; Bengtsson et al. 2007; Zhao et al. 2009). However, global simulations have a high computational cost, limiting the number of sensitivity experiments that are much needed for calibrating convection parameterizations. Consequently, they generally show variable performances in the $\mathrm{TC}$ genesis regions, with poor results in the South Pacific.

Regional atmospheric models represent a cost-effective alternative for simulating multiple seasons of TC activity. They also benefit from a geographical focus that allows better adjustments of parameterizations, and from the opportunity of controlling lateral inputs. Despite these advantages, few attempts have been made at running longterm regional TC simulations (Nguyen and Walsh 2001; Walsh et al. 2004; Knutson et al. 2007). To date, only one fine-resolution application by Walsh et al. (2004) is reported for the South Pacific, using a 30-km-mesh version of the Commonwealth Scientific and Industrial Research Organisation (CSIRO) Division of Atmospheric Research model. High-resolution atmospheric models appear to generate intense tropical cyclones, with structures that are often in remarkably good agreement with dropsonde data. However, the intensity distribution is generally distorted, with the number of both weak and extreme TCs underestimated. The climatological location of TC genesis and tracks are reasonably well simulated, although there are always some significant regional discrepancies. In particular, the South Pacific environmental conditions present a difficult task for regional TC models (Walsh et al. 2004). To improve the representation of large-scale conditions, various methods related to data assimilation can be found. While these methods may be useful to control the environmental conditions, they affect the development of intense vortices in the simulations (Bengtsson et al. 2007). A spectral nudging technique was developed by von Storch et al. (2000) to improve on this problem and constrain the large scales while allowing the model to generate smallerscale disturbances. However, TCs are mixed synopticscale-mesoscale phenomena with relatively long life spans, and selective control of their environmental conditions is difficult. Knutson et al. (2007) only use this nudging technique as a means of controlling the excessive storminess of their regional model of Atlantic TCs, not for selectively constraining the large-scale conditions.

A major objective of long-term TC simulations is to quantify the interannual variability of TC activity, identify the forcing mechanisms (whether external or internal), and estimate the degree of predictability that can be used to build cyclone warning systems. Zhao et al. (2009), when modeling the global atmosphere at $50-\mathrm{km}$ resolution, find much lower correlations between the seasonal number of observed and simulated TCs in the Pacific compared to that in the North Atlantic. Part of the discrepancy between the model and data is statistical, that is, resulting from the stochastic nature of the triggering mechanisms of TC genesis. This implies that ensemble simulations can greatly improve these correlations, especially in the North Atlantic (Vitart et al. 1997; Knutson et al. 2007; Zhao et al. 2009), even with a two-member ensemble. However, the improvements are regionally variable, and the South Pacific (as a whole) presents weak correlations even in ensemble simulations (Vitart et al. 1997; Zhao et al. 2009). Nevertheless, when subregions are considered, observational studies tend to relate cyclonic interannual variability in this region to El Niño-Southern Oscillation (ENSO). For example, significant correlations are found between the seasonal number of TCs around Australia and ENSO signals (Nicholls 1984; Evans and Allan 1992). Basher and Zheng (1995) and Kuleshov et al. (2008) also note a greater number of cyclones in the Coral Sea during La Niña than during El Niño. They also show an eastward and northward shift of cyclonic activity associated with a shift of the monsoon trough [or, equivalently, the South Pacific convergence zone (SPCZ)] during El Niño phases (see also Camargo et al. 2007a; Vincent et al. 2009).

In this paper, the Weather Research and Forecast model (WRF; Skamarock et al. 2005) is used to simulate the South Pacific TC activity and its interannual variability. Scales ranging from synoptic to mesoscale are considered so that both the environmental forcing and the mesoscale interactions needed for genesis are simulated. No assimilation or relaxation technique is used to avoid the artificial damping of storminess. We rely instead on the choice of a convective parameterization (needed for resolution larger than $5 \mathrm{~km}$ ) selected from a sensitivity experiment (section 3). The results from a 25-yr presentclimate experiment are investigated and the climatology 
of the simulated cyclonic activity is evaluated (section 4). The role of ENSO as an environmental forcing to the interannual variability of TC activity is analyzed in section 5 . Finally, the degree of stochasticity in cyclogenesis is estimated using a climatological simulation to produce a 10 -member ensemble of the same average season.

\section{Materials and methods}

\section{a. Model and configuration}

In this study, WRF version 2.2 (Skamarock et al. 2005), based on compressible, nonhydrostatic Euler equations, is used with the dynamic solver, the Advanced Research WRF (ARWRF; Skamarock and Klemp 2008). The physical parameterizations include the WRF singlemoment three-class microphysics scheme (WSM3; Hong et al. 2004), a simple cloud-interactive shortwave radiation scheme (Dudhia 1989), the Rapid Radiation Transfer Model (RRTM) for longwave radiation (Mlawer et al. 1997), the Yonsei University planetary boundary layer (YSU; Noh et al. 2003) with Monin-Obukhov surface layer parameterization, and the WRF simple five-layer (thermal diffusion) land surface model. The drag parameterization over the ocean is based on the work of Charnock (1955) and has the caveat of overestimating surface roughness at wind speed over $30-40 \mathrm{~m} \mathrm{~s}^{-1}$, with debated consequences on TC maturation (Powell et al. 2003; Moon et al. 2004). The parameterization of subgridscale convection is from the Betts-Miller-Janjic (BMJ) scheme (Janjić 1994) and was selected from a comparison with two other schemes [the Kain-Fritsch (KF) and Grell-Devenyi (GD) schemes; see section 3].

The 25-yr, present-climate simulation of TC activity in the South Pacific is designed as a dynamical downscaling product of the National Centers for Environmental Prediction (NCEP)/Department of Energy Global Reanalysis 2 (GR2; Kanamitsu et al. 2002) for the period of 1979-2004. A two-way nesting approach was chosen to extend the lateral boundaries of the model because attempts to position them near the SPCZ or intertropical convergence zone (ITCZ) introduced strong biases in the simulations (not shown). The domain is represented in Fig. 1: the coarser grid resolution is $105 \mathrm{~km}$, the inner grid resolution is $35 \mathrm{~km}$ (about $1 / 3^{\circ}$ ), and 31 vertical levels are used in both grids. The meteorological and oceanographic data used for model initialization and boundary forcing [preprocessed with the WRF preprocessing system (WPS) package] are from the 6-hourly 1979-2004 GR2. For surface forcing, GR2 includes an optimal interpolation of daily sea surface temperature (SST) analysis on a coarse $1^{\circ}$ grid, while lateral boundary forcing is imposed on the outer domain through a five-point relaxation zone.

\section{b. Effective model resolution}

The effective resolution of a model can be defined as the scale at which the model kinetic energy spectrum decays relative to the expected spectrum (Errico 1985; Skamarock 2004). The simulated January-March (JFM) mean spectrum for the 1981 model year is plotted in Fig. 2, together with the energy spectrum derived from GR2 and the $\mathrm{k}^{-5 / 3}$ Kolmogorov scaling law, describing motions at scales below $1000 \mathrm{~km}$. The break in the model slope indicates an effective resolution estimate of $155 \mathrm{~km}$, that is, about $5 \Delta x$. Effective resolution can be expected to evolve as a function of $\Delta x$, because the model filters scale with the mesh size and the ratio of effective resolution to mesh size is model dependent. The low ratio found for WRF results from its highly scale-selective implicit and explicit model filters; otherwise, that ratio is larger in most community models (Skamarock 2004).

Figure 2 shows that the GR2 forcing conditions are properly passed down to WRF because the spectra obtained for WRF and GR2 are very similar for scales larger than $3000 \mathrm{~km}$, and then small differences down to $1250 \mathrm{~km}$ are presented, which appears to be the effective resolution of GR2 in that area. The mesoscale dynamics are thus entirely handled through regional downscaling. The effective resolution of $155 \mathrm{~km}$ is finer than most mesoscale vortices formed in the SPCZ (Gray 1998) and is of the order of the smallest tropical cyclones (midget typhoons).

\section{c. Tracking methodology}

The method used here was developed by Chauvin et al. (2006) for a 50-km-resolution model. The following several criteria are used to distinguish tropical cyclones from intense midlatitude systems at each time step:

- mean sea level pressure is a local minimum;

- 850-hPa vorticity > VOR;

- maximum $850-\mathrm{hPa}$ wind speed $>\mathrm{WT}$;

- mean 700-300-hPa temperature anomaly $>$ TT;

- 300-hPa temperature anomaly $>850-\mathrm{hPa}$ temperature anomaly; and

- 850-hPa tangential wind $>300-\mathrm{hPa}$ tangential wind.

VOR, WT, and TT are threshold parameters for vorticity, wind speed and temperature anomaly respectively. Anomalies are defined as the difference between the system and its environment [both areas are defined using the radius of the maximum radial pressure gradient; see Chauvin et al. (2006)]. The tracks are then constructed by an iterative process that links the points where the criteria are satisfied; tracks shorter than 1.5 day are eliminated. As a final step, all selection criteria except the $850-\mathrm{hPa}$ vorticity $>$ VOR are removed so that the tracks are 


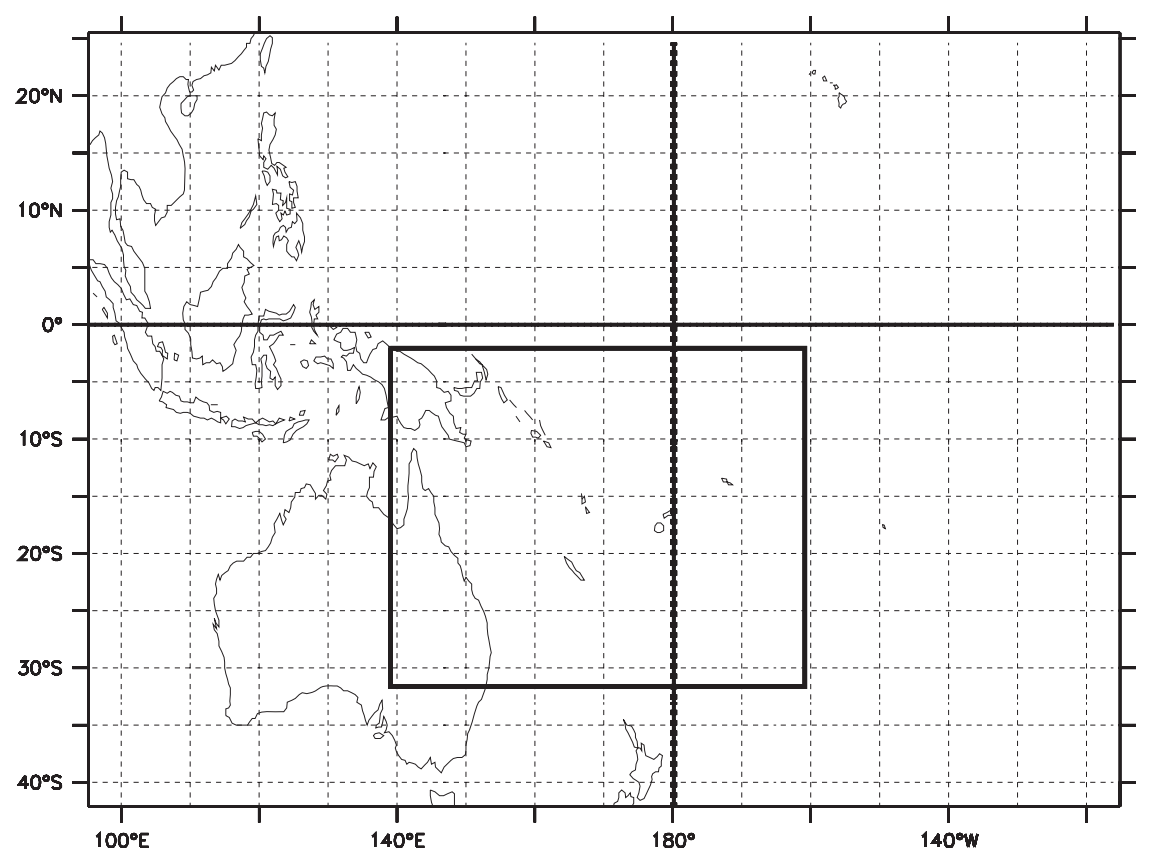

FIG. 1. Domain of integration. The black box represents the inner domain, and the map boundaries are those of the outer domain. Date line, equator, and coasts are indicated (thick black lines).

completed both backward and forward. This procedure prevents a cyclonic system whose intensity decreases below the threshold value and then increases again from being counted twice (Camargo and Zebiak 2002).

The WT threshold can be objectively determined following Walsh et al. (2007). An empirical choice of the two other thresholds was made by analyzing the first year of simulation step by step. The aim of this choice was to detect all of the significant simulated TCs (eliminating intense midlatitude vortices) rather than adjusting favorably to the real world. The analysis gives

$\mathrm{VOR}=30.10^{-5} \mathrm{~s}^{-1}, \quad \mathrm{WT}=17 \mathrm{~m} \mathrm{~s}^{-1}$ and $\quad \mathrm{TT}=3 \mathrm{~K}$.

The VOR and WT thresholds are higher than those of Chauvin et al. (2006), which is due to the higher resolution used in our analysis. VOR and TT are not chosen independently of WT because the fields of temperature anomaly, vorticity, and maximum wind speed are tightly related in TCs. However, because VOR and TT are mostly empirical values, the sensitivity of the tracking method to their choice must be checked. In Fig. 3, the surface wind speed distribution (in cyclone days) is plotted for different values of the thresholds. When removing the TT criteria (i.e., TT $=0 \mathrm{~K}$ ), there is a large increase of detected systems with low 10-m maximum wind speed, whereas the number of systems with high 10-m maximum wind speed remains almost unchanged. The opposite sensitivity is found when shifting TT to $4 \mathrm{~K}$. The vorticity threshold also has a significant impact on cyclone days for storms with low wind speed. Therefore, the strongest storms reach a stable number of detections for various threshold values (all intense TCs likely have a midtropospheric warm core and vorticity values that exceed the thresholds). On the other hand, a sufficient amount of TCs is needed to produce reliable statistical analyses. A good compromise is to only consider TCs whose surface wind speeds reach $22 \mathrm{~m} \mathrm{~s}^{-1}$ at least once (an additional criteria

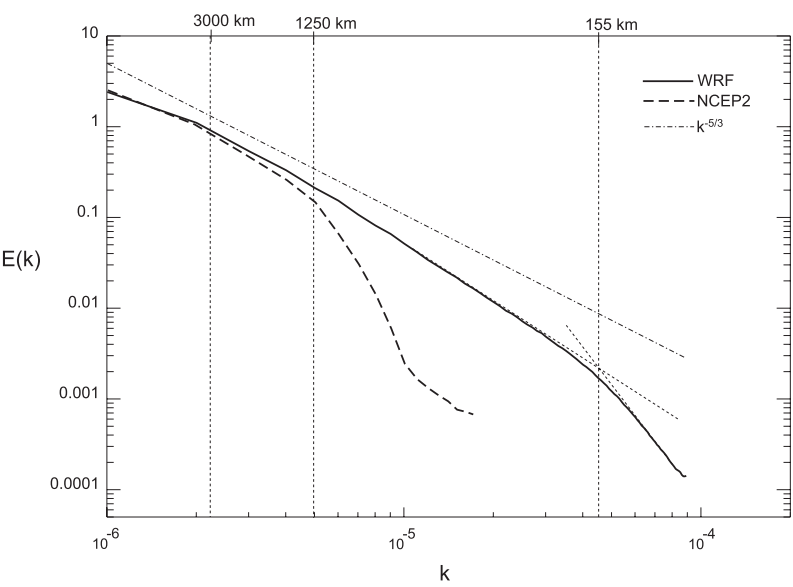

FIG. 2. JFM 1981 wavenumber spectra of simulated surface air $(10 \mathrm{~m})$ kinetic energy. $E$ is in Joules, $k$ is in $\mathrm{m}^{-1}$. 

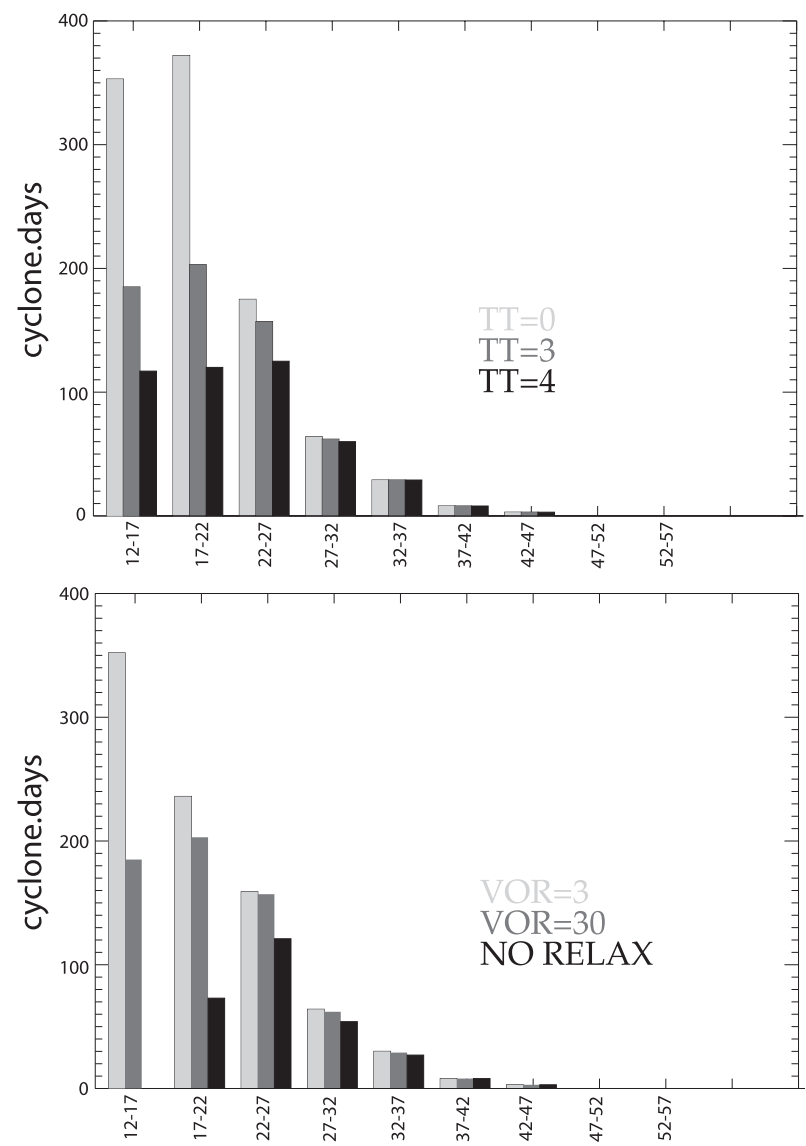

FIG. 3. Simulated 10-m wind speed distribution for the period of 1979-86 (in cyclone days versus $\mathrm{m} \mathrm{s}^{-1}$ ) according to different values of detection thresholds for temperature anomaly TT and vorticity VOR (see text for definitions). The wind speed threshold is held constant: WT $=17 \mathrm{~m} \mathrm{~s}^{-1}$. (top) TT is variable and VOR = $30.10^{-5} \mathrm{~s}^{-1}$; (bottom) VOR is variable and TT $=3 \mathrm{~K}$.

to WT); genesis location is defined as the first point along their tracks where TCs reach $17 \mathrm{~m} \mathrm{~s}^{-1}$.

\section{Convective parameterization: A sensitivity study}

MCSs found in the tropics represent 250-km areas of concentrated deep convection organized in broad mesoscale cloud clusters. Within MCSs, $50-\mathrm{km}$ areas of extreme convection can be activated and very occasionally initiate TC formation (Gray 1998). Whether they develop intense precipitation or not, MCSs are all triggered by small-scale mechanical forcings that are unresolved by our present model at $155-\mathrm{km}$ effective resolution. Therefore, we rely on parameterization of convection to produce both the large-scale heat and moisture conditions of the SPCZ and the mesoscale conditions of TC genesis.

All sensitivity tests to convection schemes are generally concerned with the evolution of individual TC events over synoptic periods; to our knowledge, none have been reported on statistical properties of free-running longterm simulations. In this section, we propose such an experiment, comparing three WRF implementations of widely used convective precipitation (CP) schemes: BMJ (Janjić 1994), KF (Kain 2004), and GD (Grell and Dévényi 2002). The BMJ scheme is a convective adjustment scheme that initially had been developed for tropical areas (Betts 1986) and successfully tested in mesoscale TC simulations (Baik et al. 1990b). Its success owes to realistic heating and moistening in the vertical resulting from a simple relaxation to observation-based reference profiles (Janjić 2000). The last two CP schemes are mass flux-type schemes, which attempt to represent updrafts/ downdrafts and entrainment/detrainment processes; they where initially developed to improve precipitation forecasts of synoptic events. The GD scheme, which uses a mass flux formulation based on averages from various closure assumptions and entrainment/detrainment rates, shows the worst results in our sensitivity tests, showing weak and patchy convection activity, numerical instability, and almost no TC formation. These results will not be overly commented on here because it is presently unclear whether its faulty behavior is a result of wrong implementation or an actual inability to handle tropical dynamics in long-term simulations.

\section{a. SPCZ sensitivity}

Simulated precipitation in the SPCZ is mainly produced by the CP scheme (see following section). It is also a good proxy for the thermal structure of the whole troposphere (Royer et al. 1998), that is, of the thermal environment of TC activity. It is therefore a natural choice for our model-data comparison. The modeled large-scale dynamics are less sensitive to the choice of CP scheme. In particular, the surface winds are reasonably close to the Quick Scatterometer (QuikSCAT) estimations, although slightly weaker (Chelton and Freilich 2005, and references therein).

The three CP schemes are evaluated against highresolution precipitation data, which are only available from January 1999 to June 2004, thereby restricting the experimental window. The main precipitation dataset is the $0.25^{\circ}$ Tropical Rainfall Measuring Mission (TRMM) product 3B-43, which combines estimates of precipitation generated from satellite-calibrated instruments (3B-42) and globally gridded rain gauge data (Adler et al. 2000). We have also used for comparison the $2.5^{\circ}$ Global Precipitation Climatology Project (GPCP) dataset (Huffman et al. 1997) because it has been more widely used in recent studies (both datasets are monthly fields). Comparing measurements of west Pacific atoll precipitation rates to TRMM and GPCP, Adler et al. (2003) found a negative bias of approximately $10 \%$ for the two datasets. They also 
noted that differences between TRMM products computed with different algorithms (based on radar and radiance measurements) can reach $30 \%$ in the heavy precipitation areas of the SPCZ.

Figure 4 shows the mean precipitation patterns observed and simulated in the western Pacific. Locations of the austral summer (JFM) ITCZ and SPCZ are represented as the meridional maximum precipitation in the Northern and Southern Hemisphere, respectively (Vincent et al. 2009). The position of the ITCZ is very similar in each model run, whereas there are large discrepancies in the SPCZ location and intensity. TRMM is very similar to GPCP in this region, but offers a better resolution, as expected. Compared with observations, the simulated SPCZ using KF extends too far south (by $5^{\circ}-10^{\circ}$ ) and has a zonal orientation east of $170^{\circ} \mathrm{E}$ rather than the classical northwest-southeast tilt. The location of the SPCZ in the BMJ experiment is much better. Regional mean SPCZ rain rates of BMJ are very similar to those of TRMM, but there are local discrepancies. Precipitation is overestimated east of the date line (by $2-4 \mathrm{~mm} \mathrm{day}^{-1}$ ), there is a bias in the SPCZ tilt east of $160^{\circ} \mathrm{W}$, and the SPCZ is located about $3^{\circ}$ too far north near the date line. Simulated precipitation over Papua New Guinea is also 2-3 times greater than in TRMM and GPCP, which can be attributed to the difficult treatment of high orography in models. Finally, precipitation is underestimated over northern Australia in all of the experiments.

We now compare the CP schemes for their impact on seasonal and interannual SPCZ variability. As shown in Lefevre et al. (2010), the migration of SPCZ location is a good indicator of seasonal variations as well as ENSO impacts on the southwest Pacific climate (through the longitudinal extension of the monsoon winds). Vincent et al. (2009) show that the main modes of SPCZ variability differ in the western and eastern parts of the SPCZ. Following this study, we define two indices to characterize the SPCZ location on either side of the date line. The western SPCZ index (SPCZ-I $\mathrm{I}_{\mathrm{W}}$ ) is constructed using the SPCZ latitude zonally averaged over $155^{\circ}-$ $175^{\circ} \mathrm{E}$, while the eastern index $\left(\mathrm{SPCZ}-\mathrm{I}_{\mathrm{E}}\right)$ is an average over $155^{\circ}-175^{\circ} \mathrm{W}$. In this section, we use monthly values of SPCZ- $\mathrm{I}_{\mathrm{W}}$, while both indices are used in section 5 (with JFM rather than monthly mean values because the eastern SPCZ is not well defined in austral winter). The comparisons between SPCZ-I $\mathrm{I}_{\mathrm{W}}$ built from precipitation data and from WRF simulations using the three CP schemes are summarized in Table 1. The standard deviations of SPCZ- $\mathrm{I}_{\mathrm{W}}$ from BMJ and $\mathrm{KF}$ are both very close to the observed ones. However, model-data correlations are much stronger using $\mathrm{BMJ}(0.64)$ than using KF (0.36). Note that these two correlation values would possibly be higher if the model could accurately simulate
Madden-Julian oscillation (MJO). The latter indeed form the other important external forcing driving SPCZ variations at the intraseasonal time scale (section 5), but for reasons yet unknown the western boundary between GR2 and WRF solutions is not transparent to the propagation of this signal (section 5).

\section{b. TC sensitivity}

In addition to a better representation of large-scale conditions, the BMJ scheme provides the only realistic

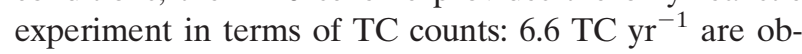
served [Joint Typhoon Warning Center (JTWC) data],

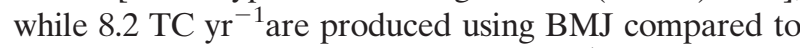
a much too large value of $25.6 \mathrm{TC} \mathrm{yr}^{-1}$ using the $\mathrm{KF}$ scheme (and only $0.2 \mathrm{TC} \mathrm{yr}^{-1}$ using the GD scheme). Because CP schemes have such a widespread impact on the preconditioning, genesis, and intensification of TCs, it is difficult at this point to interpret their various responses. Such an interpretation would require a better understanding of the schemes' trigger mechanisms and the interplay between subgrid-scale and grid-scale convection in mesoscale models. Here we can merely advance some suggestions.

Parameterization of convective transports is required in conditionally unstable situations where only intense, subgrid-scale motion and instability can trigger deep convection. However, when the air becomes saturated and grid-scale vortex motion has intensified, the resolved motion is able to sustain extreme convection, dominating the parameterization work along the eyewall (see section 4b). This model behavior, also described in Baik et al. (1990a) using the BMJ scheme, is consistent with observations that radial eddy fluxes are only dominant in the early stages of TC formation, while frictionally forced convergence would dominate later stages (see Gray 1998 for a review). The KF scheme is designed to allow more interaction between grid-scale and subgrid-scale convection, first by basing the trigger function on resolved vertical motion, and then by feeding back parameterized vertical motion to the resolved scales. As a result, this scheme is more sensitive to horizontal resolution (Gallus 1999) and may overstimulate the grid-scale contribution to deep convection at the present resolution, especially in the early stages of TC evolution. This would explain the large production of cyclones and the shift in TC distribution, from storm level to intense TCs (not shown). Note that our suggestion is reminiscent of Gray's remark (Gray 1998) that the most recent CP schemes are probably too preoccupied with catching the full intensity of TC properties in the later formation stages, because this represents the most important task for event forecasters who bypass the earlier stages using data assimilation. On the other hand, a tuning of the KF trigger function and mass flux 


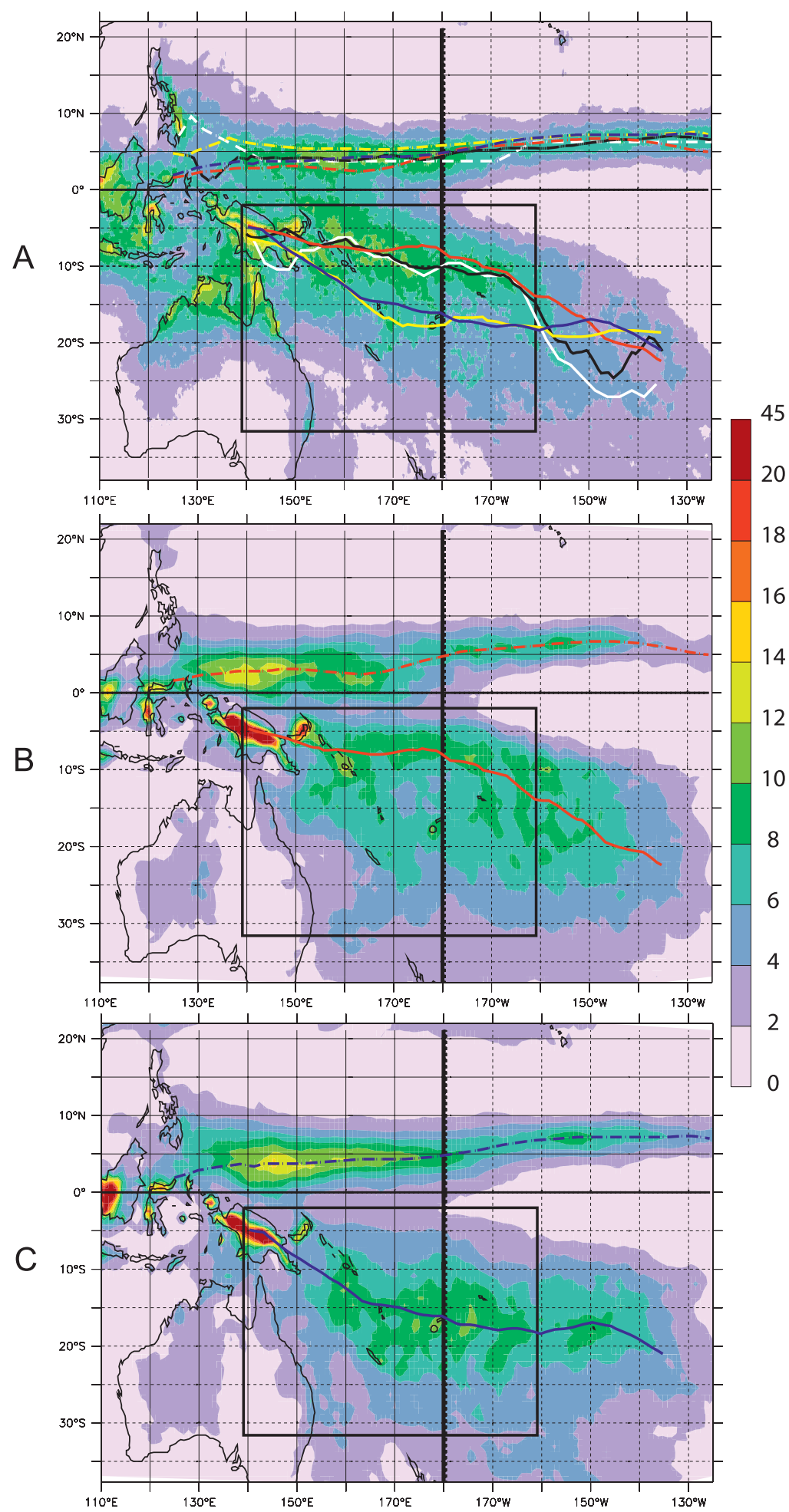

FIG. 4. Mean JFM precipitation from 2000 to $2004\left(\mathrm{~mm} \mathrm{day}^{-1}\right)$. (a) TRMM precipitation, with position of ITCZ (dashed) and SPCZ from different datasets: TRMM (black), GPCP (white), BMJ (red), KF (dark blue), and GD (yellow). (b) WRF-BMJ precipitation. (c) WRF-KF precipitation. The inner domain of integration is delimited (black box). The lines representing the SPCZ are defined as maxima of the meridionally smoothed precipitation field. 
TABLE 1. Variability of the monthly western SPCZ index; $\sigma$ is standard deviation, and $r(\mathrm{GPCP})$ and $r(\mathrm{TRMM})$ are correlation coefficients comparing each series with GPCP and TRMM, respectively. The values inside the parentheses are correlations are not significant at $95 \%$ according to the Student's $t$ test.

\begin{tabular}{clccc}
\hline \hline Index & Data & $\sigma$ & $r($ GPCP $)$ & $r($ TRMM $)$ \\
\hline \multirow{5}{*}{ SPCZ-I } & GPCP & 4.1 & 1 & 0.89 \\
& TRMM & 3.6 & 0.89 & 1 \\
& BMJ & 3.6 & 0.63 & 0.64 \\
& KF & 3.5 & 0.36 & 0.34 \\
& GD & 2.8 & $(0.20)$ & $(0.23)$ \\
\hline
\end{tabular}

formulation could probably improve the representation of transition between subgrid-scale and grid-scale convection in our experiment (Hong and Pan 1998).

It is interesting that, by shifting the pattern of largescale convergence lines with numerous mesoscale disturbances to frequent intense vortices, the excess of the intense grid-scale convection would also affect the largescale SPCZ conditions. In our BMJ experiment, parameterized convection appears to be closely linked to the resolved low-level moisture convergence, an expected relation on which earlier $\mathrm{CP}$ schemes were entirely based. In turn, convection areas produce low pressure conditions that tend to drive convergent flows. In the KF experiment, there is a shift of convection activity from the broad areas of convergence to cyclonic vortices further south, possibly explaining the southward SPCZ shift. However, a thorough investigation of SPCZ and TC sensitivity to $\mathrm{CP}$ schemes is left to further research, which should also consider the response of parameterized convection to changing resolution and ocean feedback. We conclude for now that the BMJ scheme is an obvious choice for our study and the next sections will describe in more detail the TC simulation using this parameterization.

\section{TC climatology}

The climatology of TC activity in a 25 -yr, presentclimate simulation of the South Pacific is evaluated and analyzed in this section using the available observations. Statistical analyses are performed using the tracking method described in section 2 and compared with the JTWC best tracks (Chu et al. 2002). Short-lived tracks (shorter than 1.5 days) are removed from JTWC data to be consistent with the tracking method, and because the detection of short-lived TCs in satellite data is sensitive to changes in observational systems and analysis technics (Landsea et al. 2010).

\section{a. Structural properties}

We first describe the vertical structure of simulated tropical cyclones to assess the model's ability to accurately

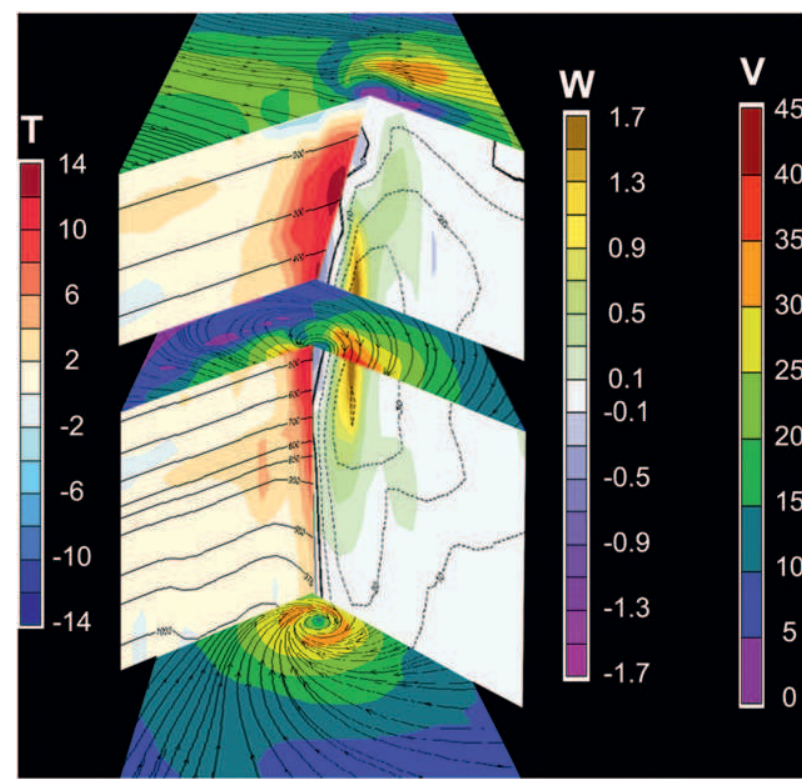

FIG. 5. Three-dimensional presentation of a modeled tropical cyclone (model date: 1800 UTC 8 Apr 1983). Horizontal maps present the horizontal wind speed $\left(V, \mathrm{~m} \mathrm{~s}^{-1}\right)$, with streamlines at $10 \mathrm{~m}, 510 \mathrm{hPa}$, and $220 \mathrm{hPa}$. (left) Warm core temperature anomalies $(T, \mathrm{~K})$ with respect to the mean temperature found $20^{\circ}$ away from the cyclone eye are shown; black contours indicate pressure (every $100 \mathrm{hPa}$ above $900 \mathrm{hPa}$, and every $25 \mathrm{hPa}$ below $950 \mathrm{hPa})$. (right) Vertical wind speed $\left(W, \mathrm{~m} \mathrm{~s}^{-1}\right)$ and tangential wind speed (dashed contours, contours every $10 \mathrm{~m} \mathrm{~s}^{-1}$ ) are presented.

reproduce the structural properties of actual systems. Several cyclonic events have been analyzed in the experiment, and a three-dimensional plot of a representative modeled TC is given in Fig. 5. This example presents a maximum $10-\mathrm{m}$ wind speed of $32 \mathrm{~m} \mathrm{~s}^{-1}$, and a maximum $700-\mathrm{hPa}$ wind speed of $45 \mathrm{~m} \mathrm{~s}^{-1}$. The maximum vertical wind speed is $1.7 \mathrm{~m} \mathrm{~s}^{-1}$ in this case and is always below $2 \mathrm{~m} \mathrm{~s}^{-1}$ in all of the other simulated cyclones. It appears to be at the lower end of the range given by airplane observations, although the latter generally concerns TCs that are more intense than those of our simulation. For example, Marks (1981) has recorded a sustained updraft velocity above $3 \mathrm{~m} \mathrm{~s}^{-1}$ over a distance of $40 \mathrm{~km}$ (about the resolution of the model), in a TC reaching a maximum wind speed $V_{\max }$ of $80 \mathrm{~m} \mathrm{~s}^{-1}$; Black et al. (1996) have observed updrafts of $4-8 \mathrm{~m} \mathrm{~s}^{-1}$ over shorter distances of $10 \mathrm{~km}$ in a TC with $V_{\max } \simeq 75 \mathrm{~m} \mathrm{~s}^{-1}$. The maximum simulated vertical ascent velocity is found between 600 and $400 \mathrm{hPa}$, which is in agreement with Jorgensen et al. (1985). There is a well-developed eye, with a maximum subsident velocity of $0.3 \mathrm{~m} \mathrm{~s}^{-1}$ between 600 and $400 \mathrm{hPa}$, which is 3 times the typical value mentioned by Emanuel (2003). Possible misrepresentation of extreme vertical velocities in the model may be 
due to missing subgrid-scale updraft velocity along the underresolved eyewall. However, we conclude that in view of observational studies, TCs vertical motions are well captured by our mesoscale model.

The maximum warm core anomaly is found between 300 and $200 \mathrm{hPa}$, where it reaches $14 \mathrm{~K}$. This value is similar to those from the literature [e.g., $16 \mathrm{~K}$ at $250 \mathrm{hPa}$ observed with dropsondes by Hawkins and Imbembo (1976) in a TC reaching $V_{\max } \simeq 65 \mathrm{~m} \mathrm{~s}^{-1} ; 11 \mathrm{~K}$ at $400 \mathrm{hPa}$ observed with dropsondes by Halverson et al. (2006) in a TC reaching $V_{\max } \simeq 54 \mathrm{~m} \mathrm{~s}^{-1} ; 8 \mathrm{~K}$ in the upper troposphere of a simple hurricane model with $V_{\max } \simeq 60 \mathrm{~m} \mathrm{~s}^{-1}$ (Emanuel 1995, 2003)]. Moreover, the vertical structure of temperature anomalies (seen from the vertical cross section in Fig. 5) is very similar to that derived from satellite imagery by Kidder et al. (2000) and from aircraft dropsondes by Hawkins and Imbembo (1976).

\section{b. Precipitation}

As mentioned before, the convection in MCSs is not explicitly resolved by the model whose effective resolution is about $155 \mathrm{~km}$. It appears, however, that the parameterization of convection within these systems is able to initiate cyclogenesis. All of the model TCs are initiated by intense convective events and the mean maximum precipitation rate at genesis time (found by the tracking method) is $5.9 \mathrm{~mm} \mathrm{~h}^{-1}$ (standard deviation $\sigma=1.5 \mathrm{~mm} \mathrm{~h}^{-1}$ ). The highest values are observed at the mature stage of TC formation after tangential winds have strongly intensified and triggered intense updraft from frictionally forced convergence. At this stage, the spatial maximum of mean TC precipitation is $20 \mathrm{~mm} \mathrm{~h}^{-1}(\sigma=$ $10 \mathrm{~mm} \mathrm{~h}^{-1}$ ), with the highest values in the whole experiment exceeding $50 \mathrm{~mm} \mathrm{~h}^{-1}$. As a comparison, Kelley et al. (2004) note that rainfall adjacent to the TC eye is higher than $10 \mathrm{~mm} \mathrm{~h}^{-1}$ most of the time and is up to $100 \mathrm{~mm} \mathrm{~h}^{-1}$ in small areas (from 163 precipitation radar overflights of TCs with intensity greater than $33 \mathrm{~m} \mathrm{~s}^{-1}$ ). At the intensifying stage in the model, there is a transition from subgrid-scale convective precipitation to grid-scale precipitation, which takes off to extreme values, consistently with idealized mesoscale simulations (Baik et al. 1990a). A typical example of TC development is illustrated in Fig. 6. After the transition period between subgrid-scale and grid-scale precipitation (characterized by oscillations of grid-scale precipitation that are described in Baik et al. 1990a), grid-scale rain rate shows a sharp increase and closely follows the time evolution of low-level wind speed and vorticity. Even though parameterized convection is an absolute requirement for cyclogenesis at this resolution, extreme precipitation results from resolved updrafts, with subgrid-scale rain rates representing only one-quarter of grid-scale peak values.

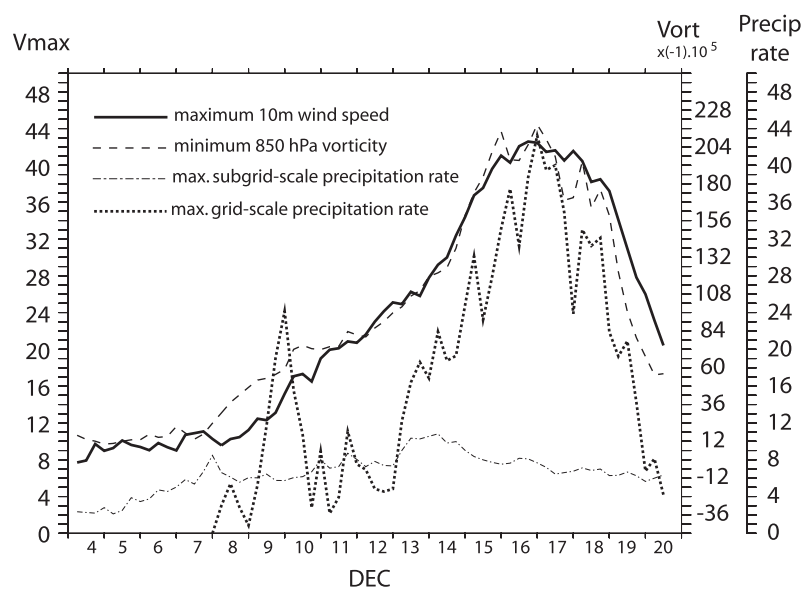

FIG. 6. Example of the evolution of a modeled tropical cyclone detected by automatic tracking in December of model year 1981 (thin dashed line). Wind speed $\left(\mathrm{m} \mathrm{s}^{-1}\right)$, relative vorticity $\left(\mathrm{s}^{-1}\right)$, and precipitation rate $\left(\mathrm{mm} \mathrm{h}^{-1}\right)$ are shown.

A question of interest is whether extreme TC rain rates contribute to a substantial part of tropical precipitation, that is, within the SPCZ. The SPCZ is defined here as the area where JFM 25-yr mean precipitation is higher than $6 \mathrm{~mm} \mathrm{day}^{-1}$ (Vincent et al. 2009). Results for various precipitation classes are shown in Table 2 . The classification first separates grid-scale precipitation resolved by the model from subgrid-scale precipitation parameterized in the CP scheme. Precipitation classes are further defined according to rain rates at each time step. Each convective precipitation intensity is related to a typical cloud cluster size. Cluster size in each class is given by the maximum width of a rectangle covering the largest cluster of a given class at each time step (the rectangular shape enables one to measure width rather than length of cloud bands). It shows that simulated climatological precipitation is mostly a result of broad mesoscale clusters, with $68 \%$ of JFM precipitation originating from systems with rates lower than $3 \mathrm{~mm} \mathrm{~h}^{-1}$. TCs are responsible for nearly $10 \%$

TABLE 2. Classification of modeled SPCZ precipitation in JFM, according to identified clusters of precipitation rates. Size statistics are constructed from size values estimated at each time step. The SPCZ is defined as the zone with climatological precipitation rates larger than $6 \mathrm{~mm}$ day $^{-1}$, with Papua New Guinea being excluded. Here, $\sigma$ is standard deviation.

\begin{tabular}{lrrr}
\hline \multicolumn{1}{c}{ Precipitation class } & \multicolumn{1}{c}{ Size } & \multicolumn{1}{c}{$\sigma$} & SPCZ \% \\
\hline Grid scale & $>1000 \mathrm{~km}$ & & $1.8 \%$ \\
Subgrid scale 0-1 $\mathrm{mm} \mathrm{h}^{-1}$ & $1010 \mathrm{~km}$ & $230 \mathrm{~km}$ & $25.1 \%$ \\
Subgrid scale $1-3 \mathrm{~mm} \mathrm{~h}^{-1}$ & $500 \mathrm{~km}$ & $110 \mathrm{~km}$ & $42.8 \%$ \\
Subgrid scale $3-5 \mathrm{~mm} \mathrm{~h}^{-1}$ & $280 \mathrm{~km}$ & $80 \mathrm{~km}$ & $17.6 \%$ \\
Subgrid scale $>5 \mathrm{~mm} \mathrm{~h}^{-1}$ & $200 \mathrm{~km}$ & $40 \mathrm{~km}$ & $3.2 \%$ \\
Subgrid scale cyclonic & $520 \mathrm{~km}$ & $120 \mathrm{~km}$ & $9.5 \%$ \\
\hline
\end{tabular}


of the total amount of precipitation in the climatological summer SPCZ, with a small fraction being contributed by grid-scale precipitation. This is comparable to the value of the $5 \%$ contribution of TCs to the 1999-2006 JFM rainfall in the South Pacific basin $\left(0^{\circ}-40^{\circ} \mathrm{S}, 135^{\circ} \mathrm{E}-150^{\circ} \mathrm{W}\right)$ found by Jiang and Zipser (2010) from TRMM data. The gridscale precipitation is thus responsible for the modeled TC peak values but is restricted to the eyewall region, while subgrid-scale precipitation is active over a much larger area with a classical spiral pattern. Note that TC displacements to higher latitudes (out of the cyclogenesis area) may contribute to extending the edges of the SPCZ. For example, the mean contribution of TC precipitation to simulated climatological precipitation around $20^{\circ} \mathrm{S}$ can reach $20 \%$ (not shown).

\section{c. Intensity distribution}

The distribution of 10-m wind speed of simulated cyclones is compared to the values from JTWC best tracks. The JTWC wind speed data are 1-min-average sustained wind speed, which needs to be compared with the 3-minaverage values of the model (corresponding to the model time step). Note that JTWC wind speeds are estimated from satellite imagery following Dvorak $(1975,1984)$ and that the method contains some uncertainty. In addition, wind speed data are often missing before 1983, and we thus limit our comparison to the period from 1983 to 2004. Figure 7 shows that WRF is able to generate intense tropical cyclones of up to $47 \mathrm{~m} \mathrm{~s}^{-1}$. This is approximately the maximum wind speed obtained by recent modeling studies (Knutson et al. 2007; LaRow et al. 2008; Zhao et al. 2009). This upper limit and the speed distribution are sensitive to model resolution and parameterizations. In our case, the simulated distribution exhibits too few very intense TCs and too many weaker ones (between 17 and $27 \mathrm{~m} \mathrm{~s}^{-1}$ ). The shift in distribution toward the lower end is probably due to insufficient model resolution. As reported by Emanuel and Nolan (2004), there is a nontrivial dependence of model storm intensity on horizontal resolution, even at grid spacing as small as $1 \mathrm{~km}$, because maximum wind speed near the eyewall results from both internal instability processes and external forcing. Additionally, the lack of oceanic feedback in the atmospheric model may produce a positive intensification effect on tropical storms, thus enhancing (from the lower end of the distribution) the intensity category of named cyclones.

\section{d. Seasonal cycle}

For robustness, we now only consider in both model and observations TCs whose 10-m wind speed exceeds $22 \mathrm{~m} \mathrm{~s}^{-1}$ at least once along the track. The period considered for comparison here is also 1983 to 2004.

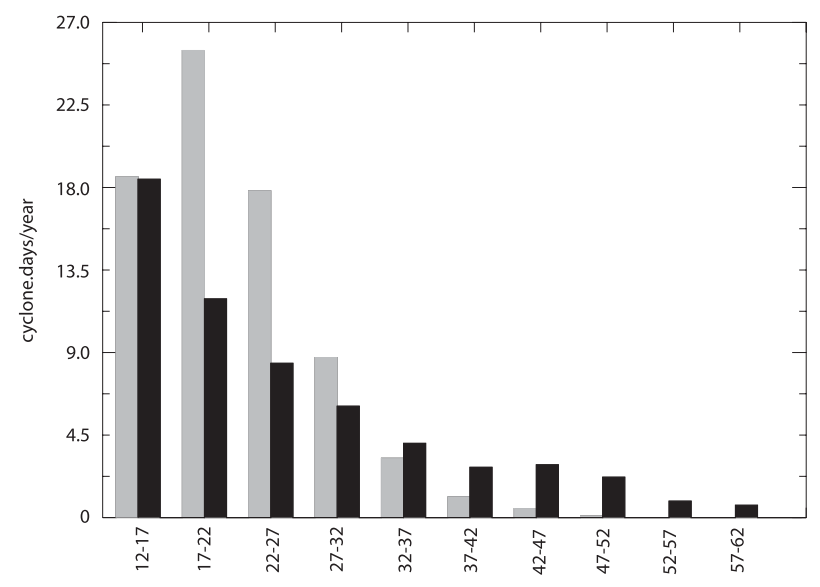

FIG. 7. Distribution of the maximum 10-m wind speed in observed cyclones (black) and in simulated cyclones (gray) between 1983 and 2004 (cyclone days versus $\mathrm{m} \mathrm{s}^{-1}$ ).

First, seasonal distribution of TC genesis and occurrence for the period 1983-2004 are plotted in Fig. 8. The general aspect of the distribution is similar in the model and observations, with most of the cyclones appearing in JFM. There are, however, some differences: $54 \%$ of the TC genesis occur in JFM in the experiment against $70 \%$ in the JTWC data. There is even a small background level of cyclogenesis persisting in the cold season [July-September (JAS)], which has never been observed (in the 1979-2004 period). To understand this peculiar result, we note that most of the JAS tropical cyclones are simulated near the northern boundary of the inner domain (not shown). An interesting feature is that among the 13 cyclones simulated in JAS between 1979 and 2004, 7 are generated in 2002 and 2003, that is, when the mean JAS SST was significantly higher than the mean 1979-2004 JAS SST in the cyclogenesis sector (i.e., 50\% more than the standard deviation in the JAS time series). The mean 2002-03 JAS vertical wind shear (between 950 and $200 \mathrm{hPa}$ ) is also lower than the mean 1979-2004 JAS value (i.e., 10\% less than the standard deviation). These large-scale anomalies, known as El Niño Modoki (Ashok et al. 2007), may have favored the simulated winter cyclogenesis (by increasing enthalpy fluxes to the atmosphere and midtropospheric humidity levels). According to the JTWC data however, the observed SST positive anomaly in winter of 2002/03 did not lead to cyclogenesis. However, the mean 2002-03 JAS precipitation rate from GPCP was higher than the 19792004 JAS mean (40\% higher than the standard deviation). This suggests that the model may be overly responsive to favorable large-scale conditions, by overrepresenting either the required trigger mechanisms of TC genesis or the convective instability processes of first and second kinds (Gray 1998). Nevertheless, this model bias remains small compared to seasonal and interannual variability. 

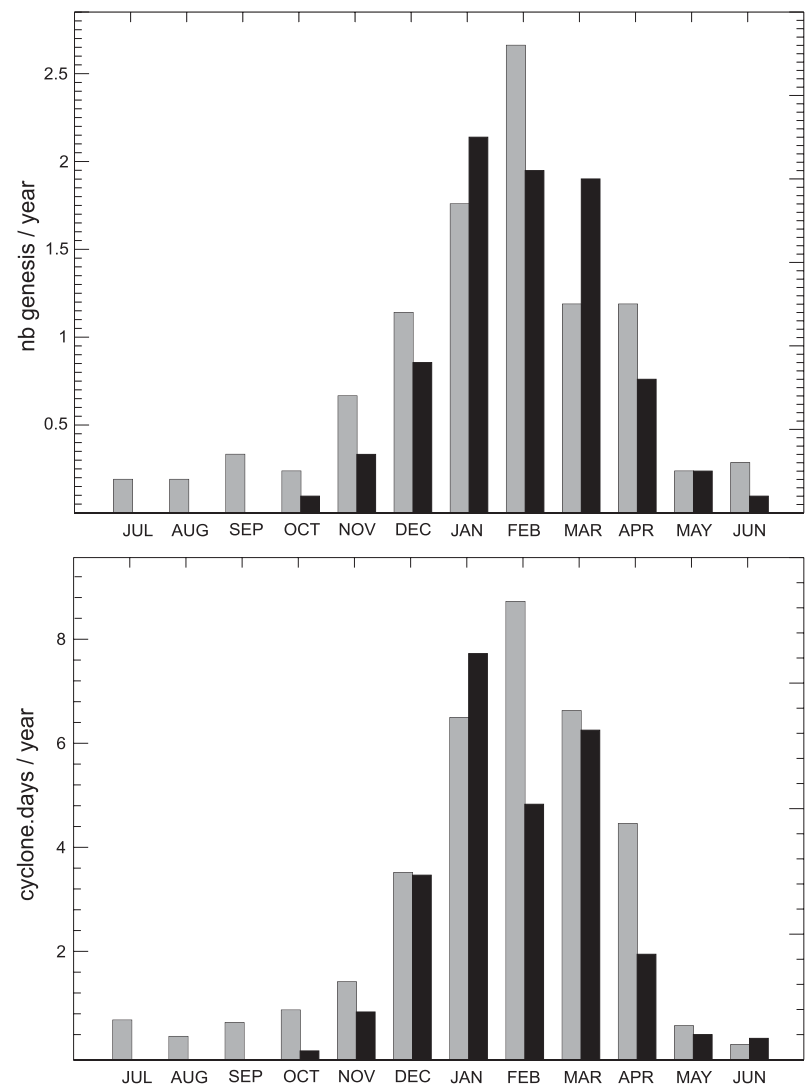

FIG. 8. Seasonal statistics of model TCs for the period between July 1983 and June 2004. (top) Model TC genesis number (cyclones reaching $22 \mathrm{~m} \mathrm{~s}^{-1}$ ). (bottom) Number of cyclone days with $10-\mathrm{m}$ wind speed higher than $22 \mathrm{~m} \mathrm{~s}^{-1}$. JTWC data (black) and simulations (gray) are shown.

\section{e. Spatial distribution}

As in Ramsay et al. (2008), probability density functions (PDFs) are used to describe the spatial distribution of both genesis and occurrence in the model and JWTC data. To generate PDFs, we compute anisotropic Gaussian functions, with an associated standard deviation in meridional and zonal directions respectively of $1^{\circ}$ and $2^{\circ}$ for occurrence, and $1.5^{\circ}$ and $3^{\circ}$ for genesis. The PDF is the normalized sum of these individual Gaussian functions associated with TC genesis or occurrence steps. This gives

$$
\iint_{\text {domain }} \operatorname{PDF}(x, y, t) d x d y=N(t)
$$

where $N(t)$ is the total number of either TC genesis or occurrence at time $t$. Figure 9 represents the 1983-2004 mean PDF for genesis and occurrence, respectively. Only tracks whose $10-\mathrm{m}$ wind speed reaches $22 \mathrm{~m} \mathrm{~s}^{-1}$ at least once are considered (see section 2c). Simulated TC genesis is defined as the first point where wind speed reaches $17 \mathrm{~m} \mathrm{~s}^{-1}$ along those tracks, while observed genesis is given by the JTWC best-track data.

The area of high TC genesis probability in the model shows notable differences with JTWC data. It appears too close to the equator (the maximum of zonal mean PDF is at $9.5^{\circ} \mathrm{S}$ in the model, but at $14.5^{\circ} \mathrm{S}$ in the observations), and there is an excess of cyclogenesis east of $170^{\circ} \mathrm{W}$ and a lack near Australia. These discrepancies are explained in the next section. The simulated occurrence is more accurate, being centered near $16^{\circ} \mathrm{S}$ both in the JTWC data and in the model, although the latter appears more dispersed with more cyclone days east of the date line. The model results in terms of intensity, and spatial and seasonal distributions of cyclonic activity are good when compared to previous modeling studies in this region (Walsh et al. 2004; Zhao et al. 2009).

\section{f. Environmental forcing}

Chauvin et al. (2006) estimate a seasonal genesis index, the convective yearly genesis parameter (CYGP; Royer et al. 1998), from the climatological conditions simulated by their high-resolution model of the North Atlantic region. They show that, despite local discrepancies, the genesis index matches the general distribution pattern of TC genesis explicitly simulated by the model. This comparison is reproduced here, and mean CYGP for the period of 1983-2004 is presented in Fig. 10. Note that our genesis index is constructed using 5-day averages of atmospheric conditions immediately prior to every genesis event. This filtering procedure is made to avoid the signature of mature TCs and highlight the large-scale environment favorable to cyclogenesis, although the difference is small from the original unfiltered CYGP.

Figure 10 shows that small areas of strong genesis index values are apparent near the Solomon and New Britain islands but these are artifacts of the high-resolution data (small-scale, high relative vorticity values should be filtered out). At larger scales, the genesis index is very similar to the PDF of genesis derived from the model (black contours in Fig. 10), with two main relative maxima at the same locations. This confirms the statement of Chauvin et al. (2006) that CYGP is a good indicator of statistical locations of simulated genesis in a high-resolution model. It also shows that the spatial distribution of cyclogenesis is controlled by the large-scale environment. Therefore, the differences with observed cyclogenesis distribution exposed in the previous section can be attributed to misrepresentation of the large-scale atmospheric conditions.

\section{TC interannual variability}

The model mean seasonal TC genesis and interannual standard deviation $\sigma_{N}$ are very comparable to the 


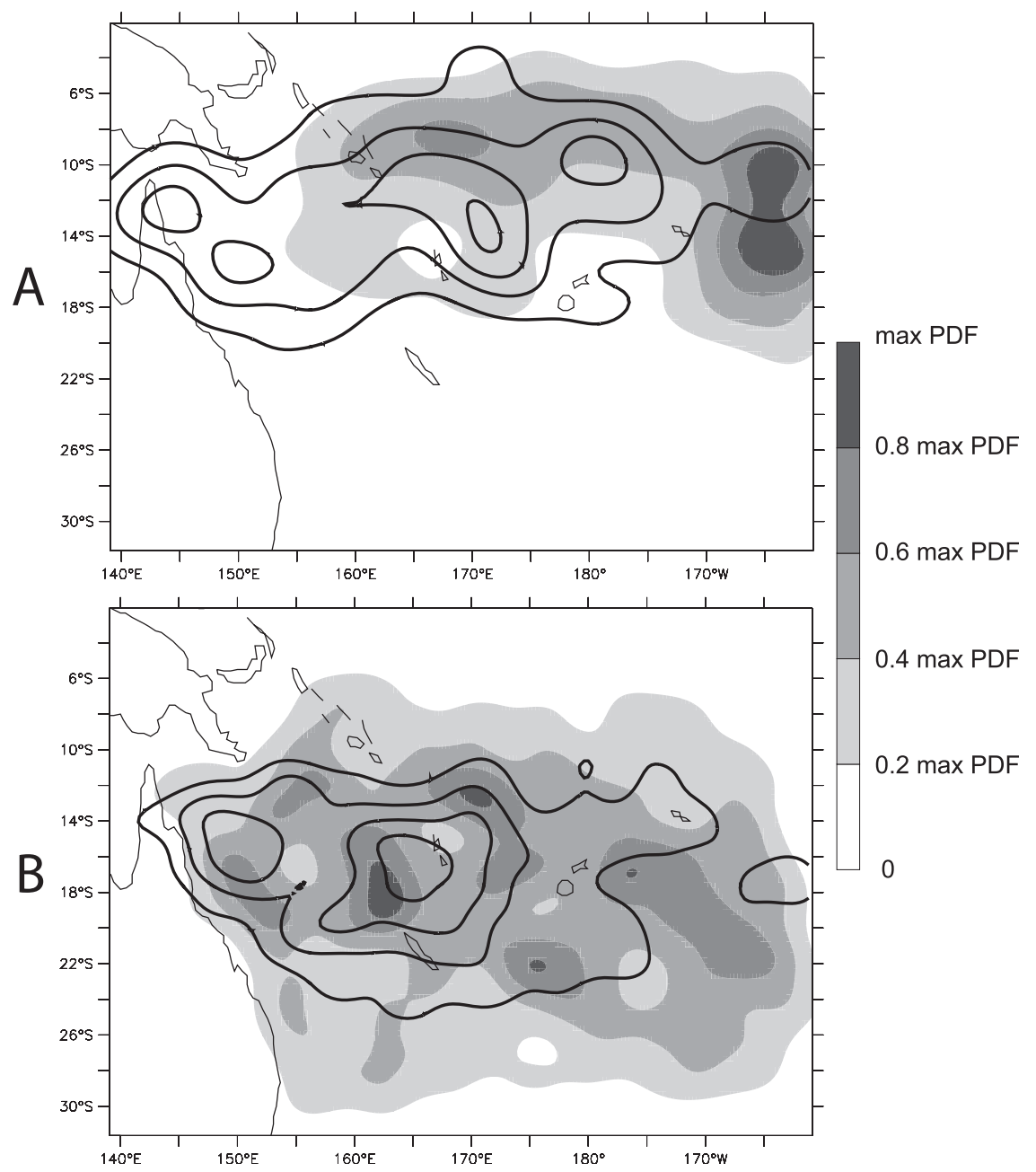

FIG. 9. PDF of TC (a) genesis and (b) occurrence for the period between July 1983 and June 2004 (cyclones reaching $22 \mathrm{~m} \mathrm{~s}^{-1}$ ). The model PDF is shown in gray and the corresponding PDF of the JTWC data is represented with black contours (interval: 0.2 max PDF).

observations: $8.2 \pm 3.5$ cyclones are produced seasonally in the model, compared with $6.6 \pm 3.0$ in the observations. Figure 11 presents the interannual variability of seasonal TC genesis averaged over the domain of integration. Interestingly, while the standard deviations are similar in the model and observations, there is no correlation between the two time series within the whole South Pacific area. In addition, the variability of TC genesis resulting from environmental control, as estimated from the CYGP genesis index $\left(\sigma_{f}=1.8\right)$, is only about half of the total value $\sigma_{N}$ (Fig. 11; note that CYGP is normalized so that the mean number of cyclogenesis estimated from the index equals the explicitly resolved genesis number). This result is insensitive to the choice of genesis index [CYGP, Gray's index (Gray 1979), or genesis potential index, GPI, from Emanuel and Nolan (2004)], as shown by Menkes et al. (2010, manuscript submitted to Climate Dyn.) using reanalysis data. We argue in this section that the lack of global correlation can be interpreted partly as compensating forced variability between southwestern and northeastern regions and partly as a product of stochasticity.

\section{a. Forced variability}

The genesis index that was previously used (CYGP) shows favorable environmental conditions in areas of deep convection. It would be expected to be maximum at the SPCZ line where convective available potential energy is maximum, but background low-level cyclonic vorticity tends to shift the most favorable conditions to the south (Fig. 10, see also Vincent et al. 2009). ${ }^{1}$

\footnotetext{
${ }^{1}$ The literature has not settled the question of whether the background low-level vorticity is a truly large-scale dynamical structure or a residual of the numerous mesoscale vortices stemming from the southern edge of the SPCZ. We leave this question open for further research.
} 


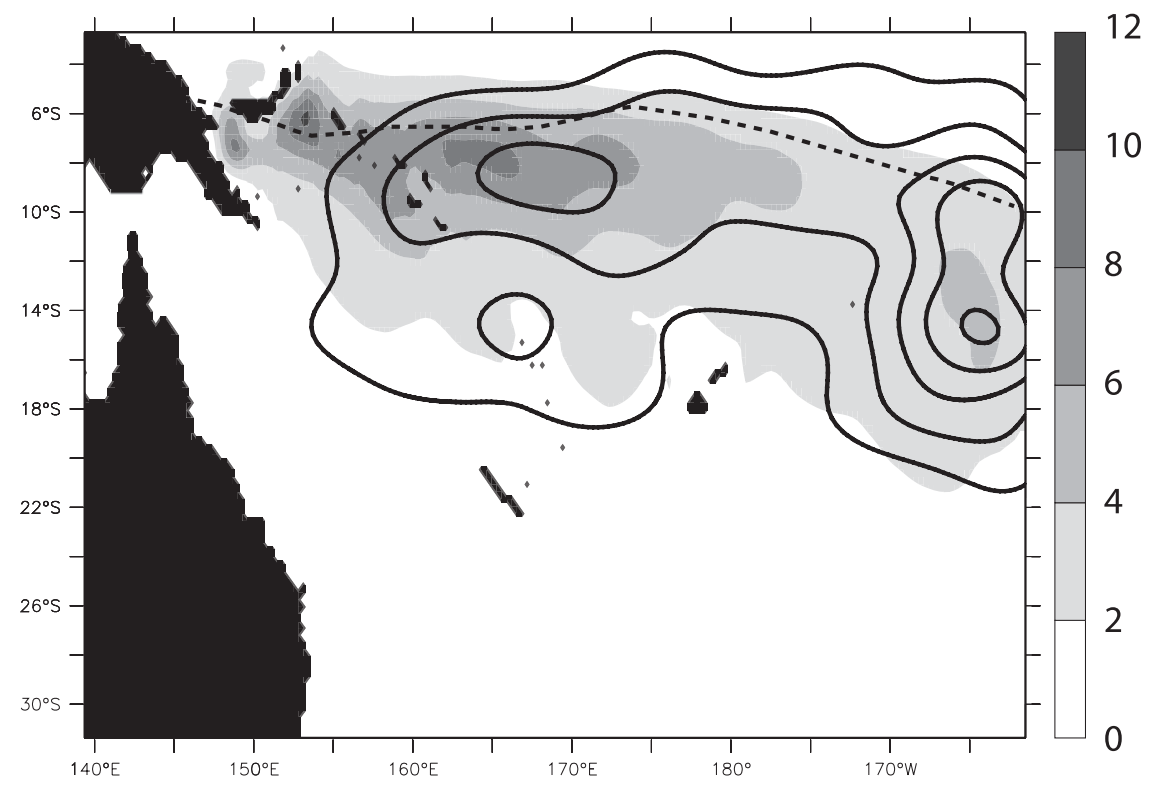

FIG. 10. Mean 1983-2004 CYGP. Land is black. The corresponding mean PDF of TC genesis is represented (thick contours, interval: 0.2 max PDF), as is the mean 1983-2004 SPCZ location (dashed thick line).

Nevertheless, the variability of the genesis index is tightly related to the SPCZ position, which therefore represents a simpler index for genesis variability in the South Pacific than CYGP (Vincent et al. 2009). The simulated SPCZ position is analyzed through a latitude index, as in section 3, but now considers both the western and eastern indices $\left(\mathrm{SPCZ}-\mathrm{I}_{\mathrm{W}}\right.$ and $\mathrm{SPCZ}-\mathrm{I}_{\mathrm{E}}$ for regions west and east of $175^{\circ} \mathrm{E}$, respectively) computed using JFM mean precipitation; seasonal and intraseasonal variations are excluded from this analysis. The simulation is again compared with GPCP precipitation data. Table 3 shows that the model SPCZ indices have significant correlations to GPCP data (a correlation of 0.71 for SPCZ- $\mathrm{I}_{\mathrm{E}}$ and 0.63 for SPCZ-I $\mathrm{I}_{\mathrm{W}}$, both of which are significant at $99 \%$ ). As in Vincent et al. (2009), four classes are defined to represent yearly changes of the SPCZ position (Fig. 12, which are closely related to ENSO phases):

- strong El Niño years,

- "positive" years (including moderate El Niño and some neutral ENSO years),

- "negative" years (including La Niña and some neutral ENSO years), and

- neutral years.

Table 4 shows error statistics (bias and root-mean-square error) of the simulated SPCZ position in the four regimes. It confirms that the modeled SPCZ in neutral and negative conditions is too zonal (with a positive bias of SPCZ- $\mathrm{I}_{\mathrm{E}}$ ). A zonal orientation of the SPCZ in nature is characteristic of strong El Niño conditions and is shown to favor TC genesis in the central Pacific (Vincent et al. 2009). This may explain our model bias toward excessive eastern cyclogenesis. In the eastern regions, the largest correlations and lowest RMSE are associated with strong El Niño conditions, that is, zonalization of the SPCZ, which occurs clearly in both the model and data.

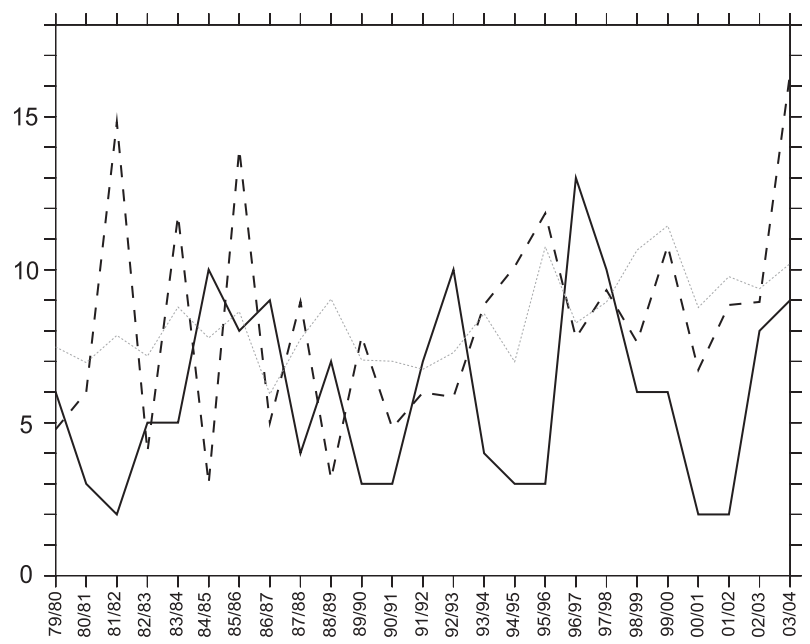

FIG. 11. Number of South Pacific TCs per season (July-June) in the JTWC data (solid line) and in the 25-yr standard experiment (dashed line). Only TCs reaching $22 \mathrm{~m} \mathrm{~s}^{-1}$ are counted. An estimation of TC genesis by the CYGP genesis index applied to the model variables is represented (dotted line). Pearson's correlation between JTWC and WRF genesis number is $r=0.30$ (not significant at the $90 \%$ level), and the correlation between WRF genesis number and CYGP is $r=0.48$ (significant at the $95 \%$ level). 
TABLE 3. Statistics of JFM SPCZ indices from 1979 to 2003; $\sigma$ is standard deviation, and $r$ (GPCP) is the correlation coefficient comparing GPCP and model (with associated significance according to the Student's $t$ test).

\begin{tabular}{llll}
\hline \hline \multicolumn{1}{c}{ Index } & Data & $\sigma$ & $r(\mathrm{GPCP})$ \\
\hline SPCZ-I & GPCP & 3.6 & 1 \\
$(\mathrm{JFM})$ & WRF & 4.4 & $0.63(99 \%)$ \\
SPCZ-I & GPCP & 6.1 & 1 \\
$(\mathrm{JFM})$ & WRF & 4.6 & $0.71(99 \%)$ \\
\hline
\end{tabular}

The spatial pattern of correlation with ENSO is given by maps of correlation coefficients between PDFs of cyclogenesis (for both the model and JTWC data) and mean November-April Niño-3.4 index (Fig. 13). These maps should be analyzed in parallel with Fig. 9, which presents model-data comparisons. The pattern of ENSO correlation for the JTWC data is consistent with a northeast shift of cyclonic activity during El Niño phases and a southwest shift (nearer to Australia) during La Niña phases (see the introduction). In the WRF experiment, there is the same pattern of correlation (with strong and significant local values), although the line separating positive and negative correlations appears to be shifted to the northeast, which is consistent with a similar bias in the SPCZ lines (Fig. 13). In both the model and data, positive correlations are located between SPCZ lines representing, respectively, strong El Niño conditions and La Niña conditions, while negative correlations are south of the SPCZ position during La Niña.

The strong interrelation between cyclogenesis and the SPCZ position may partly explain that cyclogenesis variability in the South Pacific is often poorly simulated (Zhao et al. 2009), because proper SPCZ representation is a difficult task for atmospheric models. A Newtonian nudging approach, as used by Knutson et al. (2007) in the North Atlantic, would be a way to circumvent the problem, but it would also impact the TC formation process. It has been suggested (Matthews et al. 1996) that one particular source of error in the representation of SPCZ variability may be associated with the role of the MJO, which is not captured by our model. Matthews et al. (1996) even suggest that the existence of the mean SPCZ itself may be related to the MJO. The fact that our regional model captures the mean SPCZ characteristics tends to invalidate this idea, but some of the model-data discrepancy in the interannual variability can still be attributable to missing MJO in the model. Nevertheless, in the next section, we show that the largest part of the discrepancy is due to stochasticity.

In summary, the model is able to reproduce the observed variability of TC genesis by the measure of standard deviations. The forced component of this variability
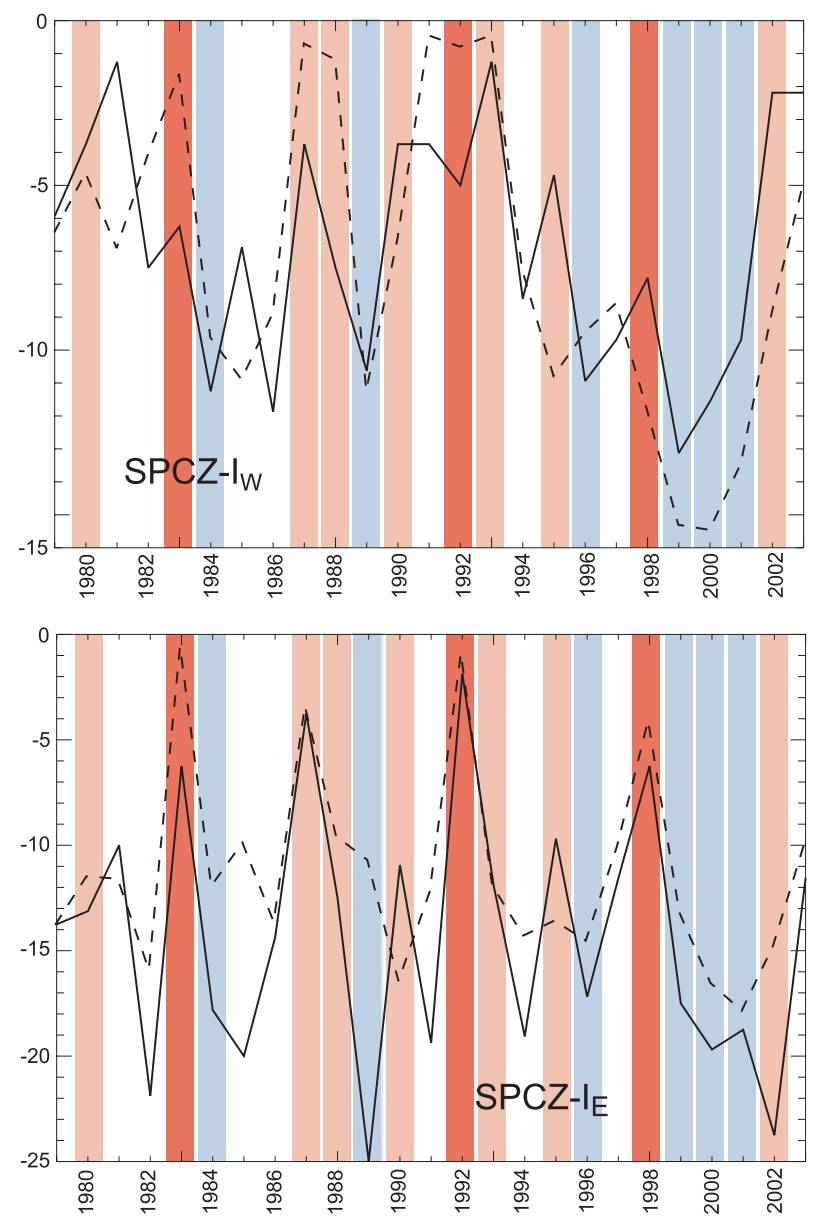

FIG. 12. SPCZ indices time series. Values are JFM means for each year; the indices computed from GPCP precipitation data (solid lines) and the 25-yr WRF experiment (dashed lines) are represented; "positive" (light red), "negative" (light blue), and strong El Niño (dark red) years are indicated.

is essentially associated with ENSO events (particularly strong El Niño phases) that impact the SPCZ position through meridional translation and zonalization. Displacement of the SPCZ position produces a corresponding displacement of cyclogenesis, which is expressed

TABLE 4. Mean bias and RMSE of the model JFM SPCZ indices compared with GPCP estimations ( ${ }^{\circ}$ latitude). A positive (negative) bias indicates that the SPCZ line is too far north (south) in the model. The categories "negative," "positive," and "strong El Niño" are shown in Fig. 12 and correspond to definitions given in Vincent et al. (2009).

\begin{tabular}{|c|c|c|c|c|c|}
\hline Index & & Neutral & Negative & Positive & $\begin{array}{c}\text { Strong } \\
\text { El Niño }\end{array}$ \\
\hline \multirow[t]{2}{*}{ SPCZ-I ${ }_{W}$} & Mean bias & 0.2 & -0.5 & -0.6 & 1.9 \\
\hline & RMSE & 3.1 & 2.2 & 4.2 & 4.1 \\
\hline \multirow[t]{2}{*}{ SPCZ-I $\mathrm{E}_{\mathrm{E}}$} & Mean bias & 3.3 & 5.1 & 0.6 & 2.8 \\
\hline & RMSE & 4.9 & 6.7 & 4.4 & 3.5 \\
\hline
\end{tabular}




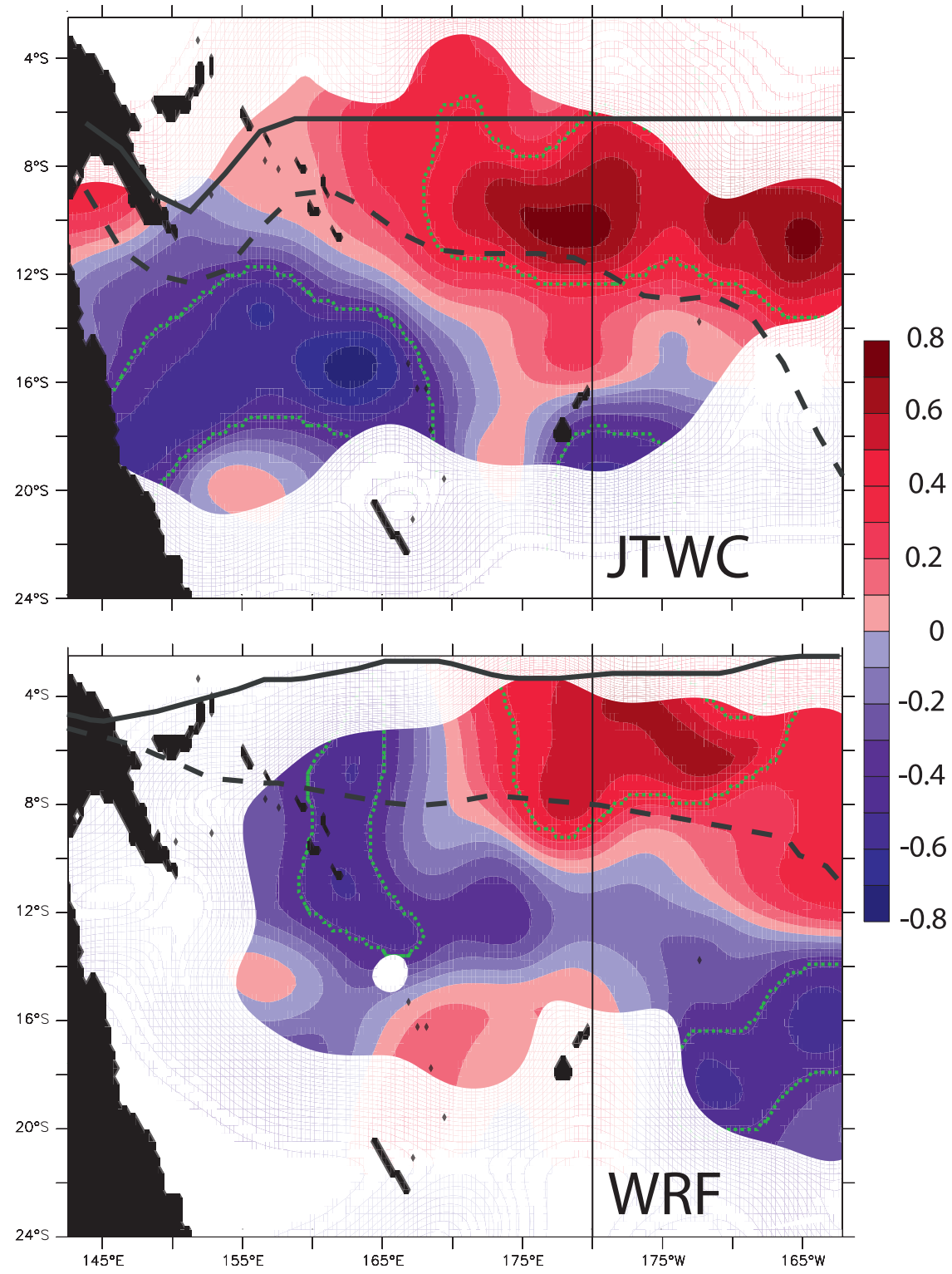

FIG. 13. Correlation coefficients between the annual mean PDF of cyclogenesis and the Niño-3.4 index averaged from November to April, for the (top) JTWC data and (bottom) WRF interannual simulation. Correlation significances of $90 \%$ according to the Student's $t$ test are represented (green dotted contours). SPCZ lines are superimposed and represent strong El Niños conditions (solid line) and La Niña conditions (dashed line).

in genesis-ENSO correlation maps as a dipole pattern. Therefore, even though local correlations can be strong, the dipole pattern results in compensating variability over the whole cyclonic activity area. The reason that the broader scale is important to consider is that TC occurrence, and hence tropical cyclone hazard, results from dispersion of TCs around the genesis location. Figure 14 shows that the dipole pattern of correlation is lost for TC occurrence as positive genesis anomalies travel southwestward (with TCs advected by the mean tropospheric flow) and mix with negative anomalies generated in the Coral Sea

\section{b. Stochasticity estimation}

Any regional model exhibits internal (intrinsic) variability that mostly depends on synoptic conditions, season, region, and model configuration (e.g., Giorgi and Bi 2000; Lucas-Picher et al. 2008). Intrinsic variability is produced by nonlinear interactions occurring at the mesoscale and synoptic scales when the domain of integration is wide 


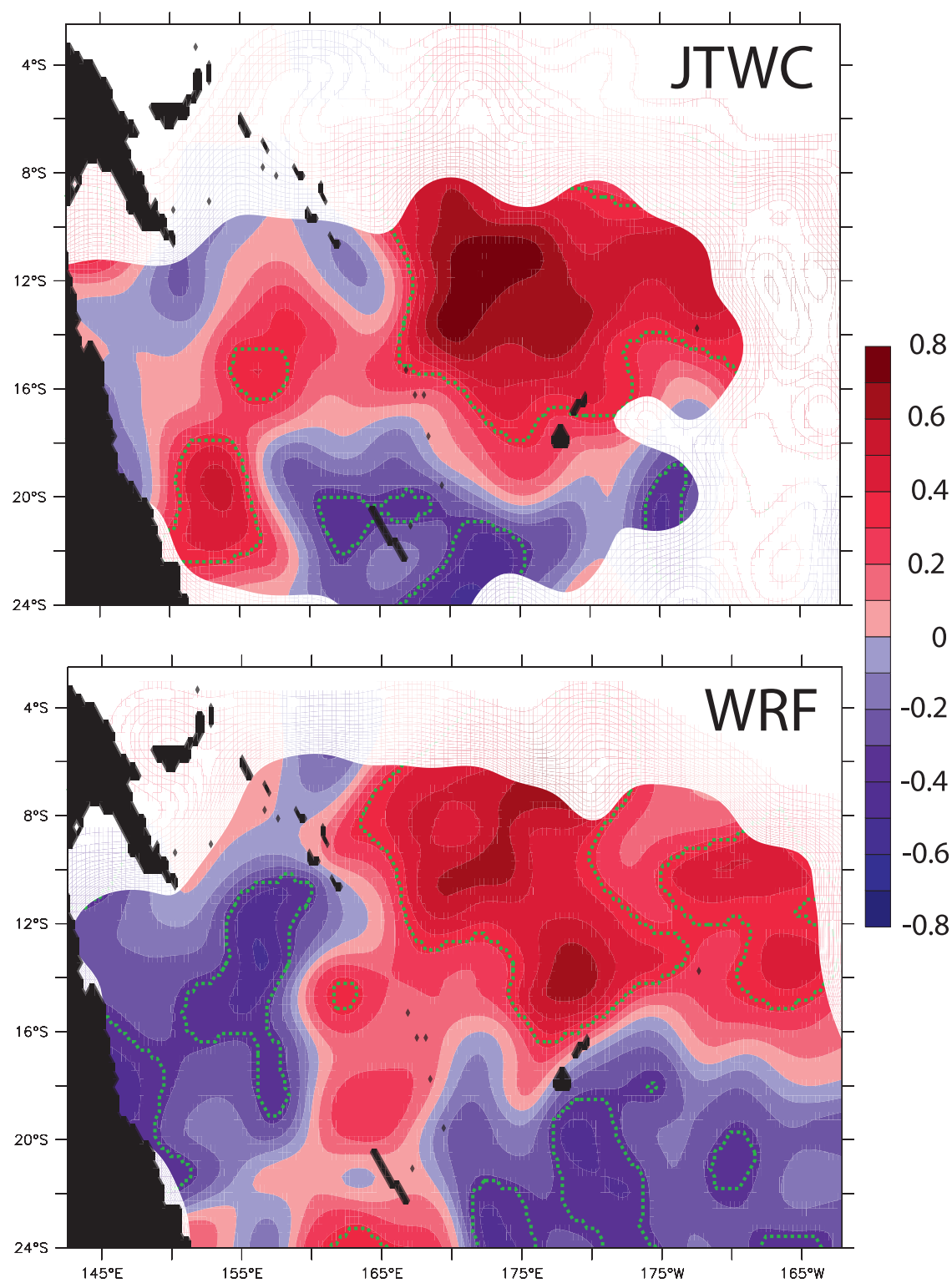

FIG. 14. Correlation coefficients between the annual mean PDF of occurrence and the Niño3.4 index averaged from November to April, for the (top) JTWC data and (bottom) WRF interannual simulation. A correlation significance of $90 \%$ according to the Student's $t$ test are represented (dotted green contours).

enough to allow for the interior dynamics to depart from the solution imposed by the lateral boundary forcing. Our objective in this section is to estimate the internal part of variability in TC activity. Error propagation rules will also provide an estimation of the part that is only forced by environmental conditions. This study thus provides a measure of seasonal predictability, that is, a measure of the stochastic error made in attempting seasonal predictions using environmental indices.

We have devised a new experiment where the regional model is forced by climatological fields. Climatological lateral and surface forcings, derived from GR2 1979-2004 means, are repeated for $10 \mathrm{yr}$. This experiment constitutes an ensemble simulation, with exactly the same forcing but different initial states every year. As shown by Alexandru et al. (2007), a 10-member ensemble is sufficient to investigate the internal variability of the model. In the climatological simulation, the mean number of TC genesis exceeding $22 \mathrm{~m} \mathrm{~s}^{-1}$ (in the inner domain) is 7.6 cyclones season $^{-1}$, that is, close to that of the standard experiment described in the previous section. The standard deviation around the mean is 1.7 cyclones season $^{-1}$ 
while the number of genesis remains within the range of 5-10 cyclones season ${ }^{-1}$.

The modeled internal variability of TC genesis may be related to both mesoscale and synoptic events. To set apart those two possibilities, the genesis index can be applied to the climatological simulation. Here again, CYGP is integrated over the domain and normalized so that the mean number of cyclogenesis estimated from the index equals the explicitly resolved genesis number. The variability of the normalized genesis index is much lower $\left(\sigma_{f}=0.4\right)$ than the variability of the number of detected cyclones $(\sigma=1.7)$ with no significant correlation between the two time series. In addition, the spatial pattern of the genesis index variance (not shown) suggests that the index variability is weak near its mean maximum, which confirms that the area of large genesis probability is the same every repeated year. We conclude that the stochastic variability of model cyclogenesis in the climatological experiment is essentially related to mesoscale processes rather than synoptic variations of environmental conditions. This result is consistent with the stochastic nature of mesoscale interactions and its suggested importance in TC genesis (Simpson et al. 1997).

Our simulations further allow description of the uncertainty propagation associated with random processes by considering the yearly TC genesis count as a product of forced and stochastic variability functions (von Storch and Zwiers 2001),

$$
\left\{\begin{array}{l}
N(t)=\phi_{s}(t) \cdot \mathrm{GI}(t) \\
\bar{N}^{t}=\overline{\mathrm{GI}}^{t}
\end{array},\right.
$$

where $\phi_{s}(t)$ is a stochastic function; $N(t)$ is the annual cyclogenesis count; and $\mathrm{GI}(t)$ is annual, spatially integrated, and normalized CYGP. We find that the values of $\ln [N(t) / \mathrm{GI}(t)]$ follow a normal distribution in both climatological and interannual experiments [using a Shapiro-Wilk test of normality with $p=0.10$; Shapiro and Wilk (1965)]. In the 25-yr interannual experiment, $\phi_{s}(t)$ follows the lognormal distribution $\log -N(\mu=-0.05 ; \sigma=$ $0.35)$ with mean and standard deviation $\bar{\phi}_{s}^{t}=0.96$ and $\sigma_{s}=0.36$. To test the usefulness of this law, $10000 \mathrm{re}$ alizations of the interannual experiment were generated using random values of $\phi_{s}$ in Eq. (2). The resulting statistics of the reconstructed TC genesis number give $8.0 \pm$ 3.3 cyclones season $^{-1}$, which is very close to the simulated values $(8.2 \pm 3.5)$. Because GI and $\phi_{s}$ are uncorrelated variables $(r=0.01)$, the rules of uncertainty propagation suggest the following statistical relations between forced and stochastic variability:

$$
\left(\frac{\sigma_{N}}{\bar{N}^{t}}\right)^{2} \simeq\left(\frac{\sigma_{f}}{\overline{\mathrm{GI}}^{t}}\right)^{2}+\left(\frac{\sigma_{s}}{{\overline{\phi_{s}}}^{t}}\right)^{2},
$$

TABLE 5. Statistics of averaged TC genesis in the South Pacific $\left(143^{\circ}-200^{\circ} \mathrm{E}\right)$ in JTWC observations, WRF simulations, and CYGP genesis index (applied to the model variables). Here, $\bar{N}^{t}$ and $\sigma_{N}$ are the annual mean and standard deviation of the number of cyclogenesis events. $\overline{\mathrm{GI}}^{t}$ and $\sigma_{f}$ are the mean and standard deviation of the estimated number of events resulting from large-scale forcing (CYGP is computed, averaged, and normalized to verify $\left.\overline{\mathrm{GI}}^{t}=\bar{N}^{t}\right) ; \bar{\phi}_{s}$ and $\sigma_{s}$ are the mean and standard deviation of the stochastic distribution estimated from Eq. (2).

\begin{tabular}{cccccccc}
\hline \hline TC statistics & $\bar{N}^{t}$ & $\sigma_{N}$ & $\sigma_{f}$ & $\sigma_{s}$ & $\left(\sigma_{N} / \bar{N}^{t}\right)^{2}$ & $\left(\sigma_{f} / \overline{\mathrm{GI}}^{t}\right)^{2}$ & $\left(\sigma_{s} \bar{\phi}_{s}{ }^{2}\right.$
\end{tabular}

where $\sigma_{N}, \sigma_{f}$, and $\sigma_{s}$ are the standard deviations of genesis number, genesis index (lowerscript $f$ stands for forcing), and stochastic distribution, respectively. It is a direct relation between the variance of genesis counts and the mean genesis number $\bar{N}^{t}$. If validated, it may be applied (in the South Pacific) to correct estimations that are based on the sole use of a genesis index. A first validation is provided by comparing in Table 5 the values of the three relative variance terms of Eq. (3). This comparison also shows that stochasticity may play a larger role $(70 \%$ of total variance) than forcing in the interannual variability of cyclogenesis, although this partition is dependent on the averaging region of interest. We checked that Eq. (3) remains valid in subregions of the southwest Pacific and that interannual forcing has a larger comparative effect in regions of strong positive or negative correlations with ENSO.

However, these estimations are affected by a degree of uncertainty associated with the capacity of the genesis index formulation to accurately represent the environmental forcing. If CYGP is accurate, we would expect to find the same stochastic distribution $\phi_{s}$ in the climatological and interannual experiments, but the standard deviation $\sigma_{s}$ is 0.22 in the climatological experiment instead of 0.36 . Even though these values are not significantly different given the small number of simulated seasons (an $F$ test does not reject the hypothesis of equal variance at the $10 \%$ level), they may suggest that stochasticity is overestimated in the interannual experiment analysis (as a result of uncertainties in the CYGP formulation), or alternatively that the climatological experiment underestimates interactions between forced and stochastic processes. In any case, we can safely conclude that stochastic variability resulting from mesoscale interactions in the South Pacific is never negligible and may even be very large. 


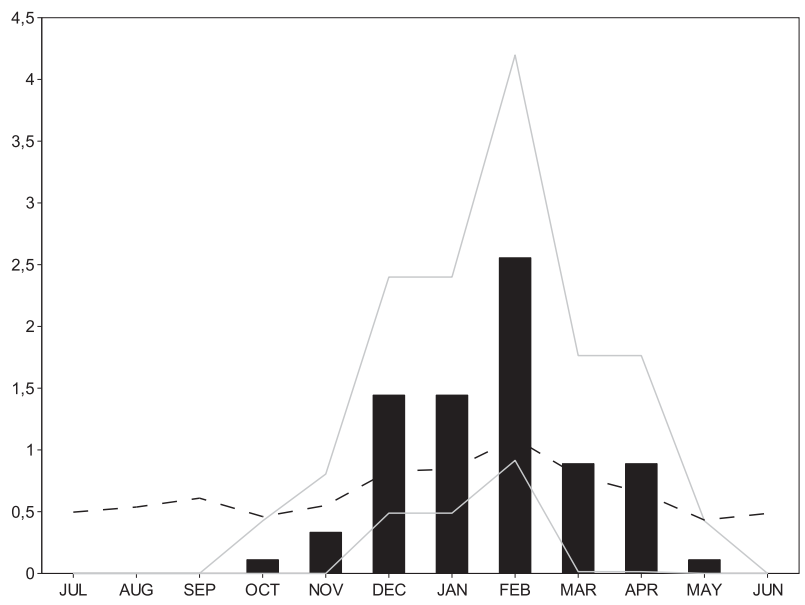

FIG. 15. Mean monthly number $N$ of model TC genesis in the 10 -yr climatological experiment. Gray curves indicate $N+\sigma$ and $N-\sigma$, where $\sigma$ is the standard deviation for each month. Monthly values of CYGP computed using the 10-yr climatological experiment are shown (dashed curve).

Note finally that the seasonal distribution of cyclogenesis in the climatological experiment (shown in Fig. 15) presents no genesis in winter, as opposed to the $25-\mathrm{yr}$ interannual experiment (Fig. 8). This confirms that winter genesis in the interannual experiment only results from anomalous seasonal conditions, not from stochastic behavior under mean forcing conditions (section 4d). In addition, Fig. 15 shows that the monthly ratio $\sigma_{N} / N$ is almost constant during the months of strong cyclonic activity [December-February (DJF) here], which is consistent with Eq. (3) using monthly rather than annual parameters ( $\sigma_{f}$ is small). However, during the months with fewer cyclogenesis, Eq. (3) is not satisfied. This analysis thus suggests that Eq. (2) cannot be straightforwardly applied to describe seasonal variations of cyclogenesis resulting from the poor representation of the seasonal cycle of genesis indices (Fig. 15; see also Menkes et al. 2010, manuscript submitted to Climate Dyn.).

\section{c. Mesoscale interactions}

We now suggest some mechanisms by which mesoscale interactions in the model can produce stochasticity in TC activity. These simulated mesoscale interactions are compared to some observations reported in the literature.

A step-by-step examination of individual TC formations along all seasons of both the interannual and climatological simulations shows that mesoscale interactions are ubiquitous in the cyclogenesis area. These interactions are various but appear to follow a pattern. Multiple mesoscale vortices and associated cloud clusters are first produced along SPCZ lines of strengthened convergence (mostly around $10^{\circ} \mathrm{S}$ ). Interaction then occurs, involving two or even three vortices of various intensities. Our modeled vortex interactions appear to span the different regimes of known classification (Dritschel 1995), from elastic interaction to partial/complete straining and partial/ complete merger. On the occasions of complete mergers, two vortices of unequal sizes can merge into one larger vortex and form an intense TC, as illustrated in Fig. 16. The process apparent in this example seems to be in good agreement with potential vorticity theories of vortex interaction and formation of spiral bands in TCs (Guinn and Schubert 1993). It includes mutual cyclonic rotation, followed by the weaker vortex being elongated (by shearing strain) and wrapped cyclonically around the stronger one to become a spiral band. During this process, the weaker vortex provides additional latent heat to the stronger one, fueling it at its own expense (it is destroyed by the intense middle-level flow of the stronger vortex). The merger takes about 3 days to proceed and the final merger occurs at a distance of about $500 \mathrm{~km}$, closely resembling the merger, described in Kuo et al. (2000), of TCs Zeb and Alex (October 1998 in the North Pacific). Note that not all vortex interactions in the model lead to vortex growth, and most cyclones do not appear to result from merger, but at some point they are almost all interacting with the mesoscale flow.

The interaction of formed TCs with mesoscale features is also responsible for the significant variability in $\mathrm{TC}$ tracks (Holland and Lander 1993). Combined with random variations of genesis location, it may increase the dispersion effect on TC occurrence. This is illustrated in Fig. 17, showing TC tracks in different years of the climatological simulation. This argument reiterates the point previously made in section 5 a that the relation between ENSO and cyclonic activity is degraded when considering TC occurrence rather than genesis.

\section{Conclusions}

In this paper, we have examined the ability of a regional mesoscale model (WRF) at $1 / 3^{\circ}$ resolution to simulate the statistics of tropical cyclone activity in the present climate of the South Pacific. A preliminary objective was to properly simulate the climatological conditions of TC genesis, in particular the characteristics defining the South Pacific convergence zone. The results found were mostly sensitive to the choice of cumulus parameterization, because the largest part of precipitable convection is still subgrid scale at this resolution. The Betts-Miller-Janjic convective adjustment scheme was selected because it clearly shows the best ability to represent the SPCZ position and associated precipitation rates compared with two mass flux convection schemes. The interannual variability of the SPCZ is also mostly 

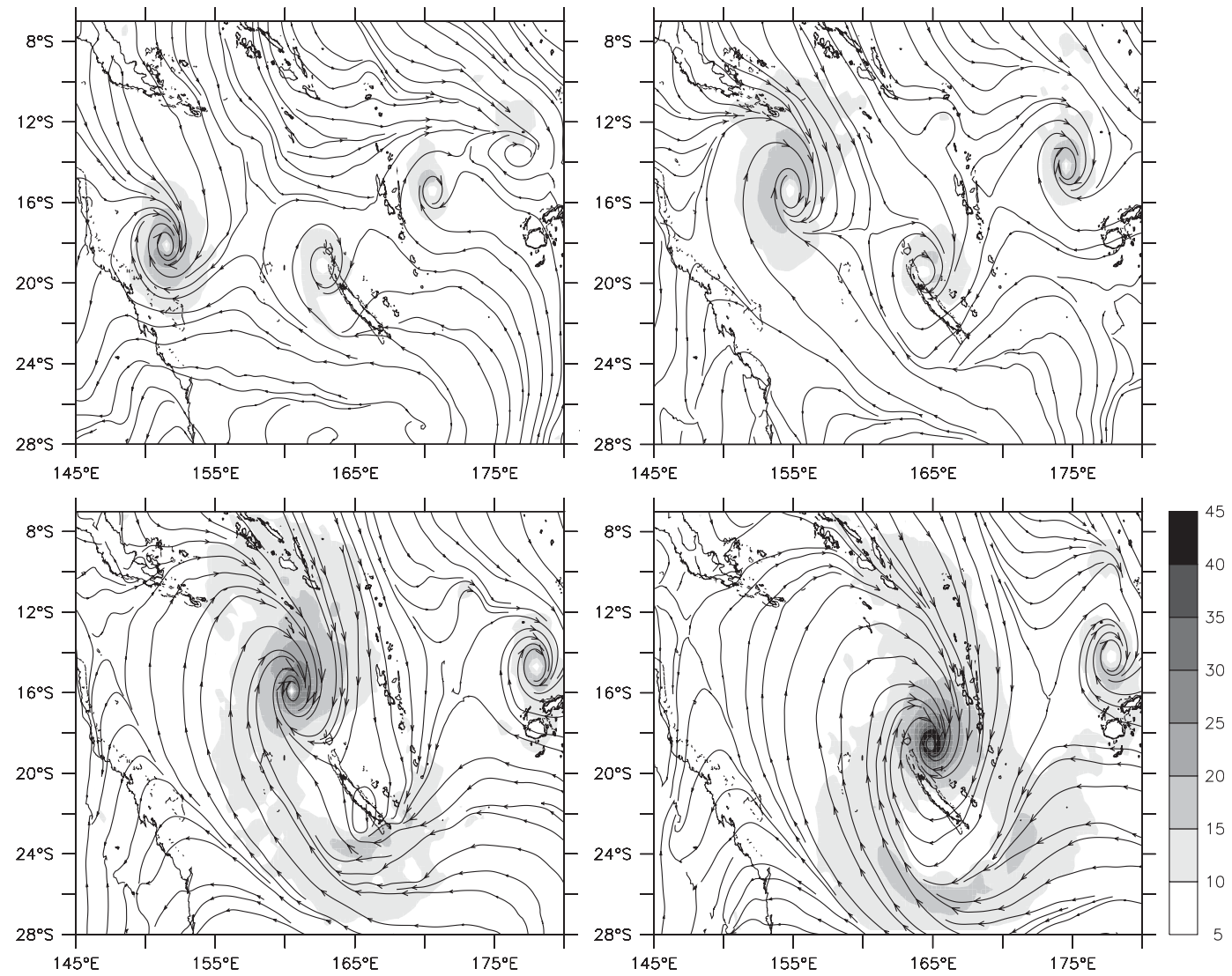

FIG. 16. Simulated TC formation by vortex merging in the Coral Sea. A 4-day event of the model year 1986, on 8, 9 , 11 , and 12 February, respectively, is shown. Low-level wind (at $925 \mathrm{hPa}$ ) is shown as streamlines and colors $\left(\mathrm{m} \mathrm{s}^{-1}\right)$.

captured by the model, particularly its zonalization during strong El Niño events.

With a grid scale of $35 \mathrm{~km}(155-\mathrm{km}$ effective resolution), we have shown that WRF is able to reproduce a wide range of mesoscale convective systems. Cloud clusters with horizontal scales around $500 \mathrm{~km}$ constitute the bulk of precipitation in the SPCZ, but the most intense precipitation systems have only small residual effects. Tropical cyclones in particular represent only $10 \%$ of the mean SPCZ precipitation. Yet they grow from the most intense systems formed along SPCZ lines of strengthened convergence (generally around $10^{\circ} \mathrm{S}$ ) and sometimes develop into hurricanes of up to $45 \mathrm{~m} \mathrm{~s}^{-1}$ (maximum 10-m wind speed). As in previous numerical studies (e.g., Knutson et al. 2007; LaRow et al. 2008; Zhao et al. 2009), the maximum intensity of observed hurricanes is not captured by the model (Saffir-Simpson category 5 includes TCs of over $70 \mathrm{~m} \mathrm{~s}^{-1}$ sustained wind speed). This can be partly explained by the known relation between TC intensity and model resolution, but also by the approximations of physical parameterizations. The threedimensional structure of simulated tropical cyclones is nevertheless in remarkable agreement with dropsondes and satellite observations. The mean seasonal and spatial distributions of $\mathrm{TC}$ genesis and occurrence are in reasonably good agreement with the JTWC data. We note, however, that the spatial pattern of TC genesis is shifted to the northeast due to a similar bias in the environmental forcing. Over the whole genesis area, the annual TC count and interannual standard deviation are also very comparable to observations: $8.2 \pm 3.5$ cyclones are produced seasonally in the model, compared with $6.6 \pm 3.0$ in the JTWC observations. The model is thus able to reproduce the observed range of variability, some of it associated with ENSO forcing (particularly strong El Niño phases). ENSO events impact the SPCZ position through meridional translation and zonalization. Displacement of the SPCZ position leads to a corresponding displacement of cyclogenesis, which is expressed in correlation maps as a dipole pattern. Therefore, even though local correlations can be strong, the dipole pattern results in weaker regional correlations resulting from compensating variability.

Large-scale forcing is not the only process driving interannual variations of TC genesis in the South Pacific; more than half of it may be due to stochastic processes. 

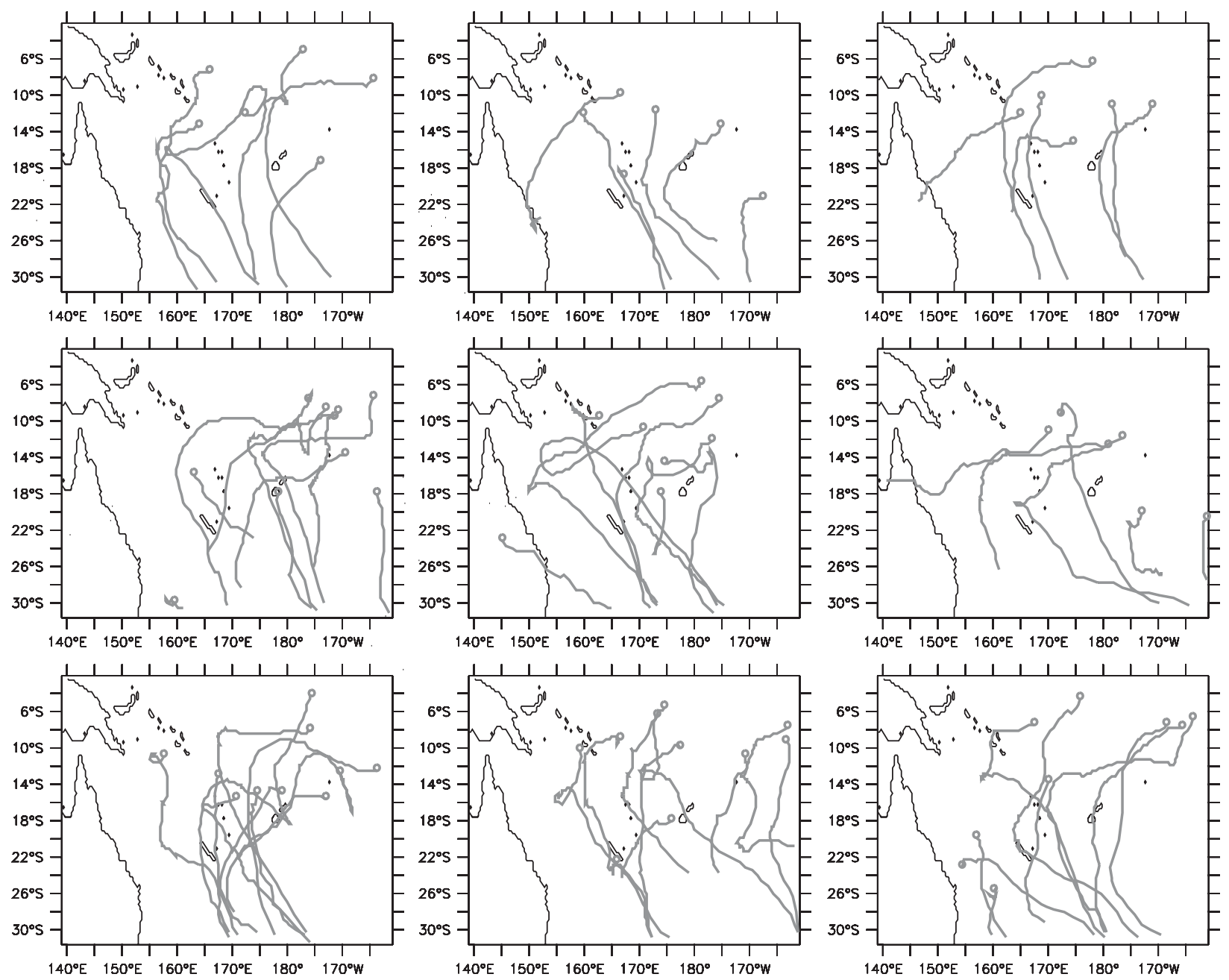

FIG. 17. TC tracks (thick gray lines, the circles represent the beginning of the tracks) computed for each year (from July to June) of the 10 -yr climatological experiment. Coastlines are in black.

This estimation of intrinsic variability was permitted by a 10-yr climatological simulation that operated as a 10-member ensemble simulation of an averaged year with differing initialization values. It shows that a standard deviation of 1.7 cyclones $\mathrm{yr}^{-1}$ emerges from the internal variability of the model (compared to the total of 3.5 cyclones $\mathrm{yr}^{-1}$ ). Because the internal variability of the regional environment (not transmitted through the lateral boundaries) is low, the internal variability of cyclogenesis in the ensemble simulation appears essentially related to mesoscale interactions. We are aware that the use of subgrid-scale convective parameterization may lead to misrepresentation (over- or underrepresentation depending on the $\mathrm{CP}$ scheme) of small-scale random processes needed for triggering TC genesis. As an example of overrepresentation, our model appears excessively sensitive to favorable winter anomalies of environmental conditions, producing occasional winter TC events. Nevertheless, assuming that mesoscale vortex interactions (at scales larger than $200 \mathrm{~km}$ ) constitute a large source of internal variability (Simpson et al. 1997), our explicit resolution of these ubiquitous stochastic processes, from elastic interactions to merger, gives us some confidence that the model has captured an interesting part of reality.

A possible way to cope with intrinsic variability and the associated loss of predictability is to perform ensemble simulations, although the efficiency of ensemble predictions depends on the region of application. Using a fourmember ensemble simulation with a $50-\mathrm{km}$ resolution model, Zhao et al. (2009) find a correlation with observed TC genesis of 0.8 in the North Atlantic, but only 0.6 in the east Pacific, 0.5 in the west Pacific, and even 0.3 in the South Pacific. Estimation of interannual variability from genesis indices are consistent with these results, showing the best predictability in the Atlantic basin (Camargo and Sobel 2004; Menkes et al. 2010, manuscript submitted to 
Climate Dyn.), where forced variability seems to be dominant. In our study, comparison of TC genesis from direct TC counts and from estimates using a genesis index (Table 5) shows that the low standard deviation of yearly genesis index in the South Pacific may be due to comparatively large stochastic variability and less so to index failure. In this case, a correction to genesis indices can be applied using stochastic distributions estimated from mesoscale models. However, the limit of this method is dependent on the genesis index accuracy to represent the environmental forcing.

Finally, we can make three concluding remarks concerning the benefit of ensemble simulations for improving predictability in the South Pacific:

- If stochastic variability in the real world is similar to that of the model, then it also constitutes a large part of its interannual variability. Ensemble simulations can only reduce the model uncertainties, not the observed ones. Therefore, the latter will form a limit to predictability, independent from model improvements.

- Correlations are greater in local rather than regional areas. Therefore, the efficiency of ensemble simulations may well be locally high but regionally low. The reason that the broader scale is important to consider is because TC occurrence, and hence landing and population vulnerability, results from dispersion of TCs around genesis location. TCs move approximately with the barotropic background airflow and their tracks are affected by a number of phenomena, including synoptic variability and chaotic advection, in which mesoscale interactions can act as a trigger mechanism. The dispersion of TC tracks, amplifying the uncertainty of genesis location, can thus explain the degraded relation between ENSO and TC activity when considering occurrence rather than genesis. Nevertheless, TC occurrence remains correlated to the interannual forcing at some locations, especially in eastern regions, that is, away from high cyclonic activity (Basher and Zheng 1995; Kuleshov et al. 2008).

- Model errors constitute another source of uncertainty and are difficult to assess. Ensemble simulations with varying model parameters may lower the model error, but cannot account for missing physics. Sensitivity to convective parameterization, model resolution, ocean feedback, and the wave interface under extreme events are all important areas of future research. However, we were able to reduce a major component of model errors by selecting the configuration and parameterizations that allowed a more realistic representation of SPCZ forcing and statistical TC counts than thatpreviously shown (Vitart et al. 1997; Walsh et al. 2004; Zhao et al. 2009). This result and indications that mesoscale interactions are realistic in the model gives us confidence that the present work contributes new and robust elements to the understanding of TC activity in the region.

Acknowledgments. We acknowledge support from the French Agence Nationale de la Recherche (ANR Project Cyclones and Climate). The authors wish to thank Jean-François Royer and three anonymous reviewers for their careful review.

\section{REFERENCES}

Adler, R. F., G. J. Huffman, D. T. Bolvin, S. Curtis, and E. J. Nelkin, 2000: Tropical rainfall distributions determined using TRMM combined with other satellite and rain gauge information. J. Appl. Meteor., 39, 2007-2023.

, C. Kummerow, D. Bolvin, S. Curtis, and C. Kidd, 2003: Status of TRMM Monthly Estimates of Tropical Precipitation. Meteor. Monogr., No. 51, Amer. Meteor. Soc., 223 pp.

Alexandru, A., R. de Elia, and R. Laprise, 2007: Internal variability in regional climate downscaling at the seasonal scale. Mon. Wea. Rev., 135, 3221-3238.

Ashok, K., S. K. Behera, S. A. Rao, H. Weng, and T. Yamagata, 2007: El Niño Modoki and its possible teleconnection. J. Geophys. Res., 112, C11007, doi:10.1029/2006JC003798.

Baik, J.-J., M. DeMaria, and S. Raman, 1990a: Tropical cyclone simulations with the Betts convective adjustment scheme. Part I: Model description and control simulation. Mon. Wea. Rev., 118, 513-528.

,$- \ldots$, and $-1990 \mathrm{~b}$ : Tropical cyclone simulations with the Betts convective adjustment scheme. Part II: Sensitivity experiments. Mon. Wea. Rev., 118, 529-541.

Basher, R. E., and X. Zheng, 1995: Tropical cyclones in the southwest Pacific: Spatial patterns and relationships to Southern Oscillation and sea surface temperature. J. Climate, 8, 12491260.

Bengtsson, L., M. Botzet, and M. Esch, 1996: Will greenhouse gasinduced warming over the next 50 years lead to higher frequency and greater intensity of hurricanes? Tellus, 48A, 57-73.

_ K. I. Hodges, and M. Esch, 2007: Tropical cyclones in a T159 resolution global climate model: Comparison with observations and re-analyses. Tellus, 59A, 396-416.

Betts, A. K., 1986: A new convective adjustment scheme. Part I: Observational and theoretical basis. Quart. J. Roy. Meteor. Soc., 112, 677-691.

Black, M. L., R. W. Burpee, and F. D. Marks Jr., 1996: Vertical motion characteristics of tropical cyclones determined with airborne Doppler radial velocities. J. Atmos. Sci., 53, 11831197.

Camargo, S. J., and S. E. Zebiak, 2002: Improving the detection and tracking of tropical cyclones in atmospheric general circulation models. Wea. Forecasting, 17, 1152-1162.

— , and A. H. Sobel, 2004: Formation of tropical storms in an atmospheric general circulation model. Tellus, 56A, 56-67.

, A. G. Barnston, and S. E. Zebiak, 2005: A statistical assessment of tropical cyclone activity in atmospheric general circulation models. Tellus, 57A, 589-604.

, K. A. Emanuel, and A. H. Sobel, 2007a: Use of a genesis potential index to diagnose ENSO effects on tropical cyclone genesis. J. Climate, 20, 4819-4834. 
A. H. Sobel, A. G. Barnston, and K. A. Emanuel, 2007b: Tropical cyclone genesis potential index in climate models. Tellus, 59A, 428-443.

Charnock, M., 1955: Wind stress on a water surface. Quart. J. Roy. Meteor. Soc., 1, 639-640.

Chauvin, F., J.-F. Royer, and D. Deque, 2006: Response of hurricanetype vortices to global warming as simulated by ARPEGEClimat at high resolution. Climate Dyn., 27, 377-399.

Chelton, D., and M. Freilich, 2005: Scatterometer-based assessment of $10-\mathrm{m}$ wind analyses from the operational ECMWF and NCEP numerical weather prediction models. Mon. Wea. Rev., 133, 409-429.

Chu, J. H., C. R. Sampson, A. S. Levine, and E. Fukada, 2002: The Joint Typhoon Warning Center tropical cyclone best-tracks, 1945-2000. Naval Research Laboratory Rep. NRL/MR/754002-16. [Available online at http://www.usno.navy.mil/NOOC/ nmfc-ph/RSS/jtwc/best_tracks/TC_bt_report.html.]

Dritschel, D. G., 1995: A general theory for two-dimensional vortex interactions. J. Fluid Mech., 293, 269-303.

Dudhia, J., 1989: Numerical study of convection observed during the Winter Monsoon Experiment using a mesoscale two-dimensional model. J. Atmos. Sci., 46, 3077-3107.

Dvorak, V. F., 1975: Tropical cyclone intensity analysis and forecasting from satellite imagery. Mon. Wea. Rev., 103, 420-430. , 1984: Tropical cyclone intensity analysis using satellite data. NESDIS Tech. Rep. 11, NOAA Tech. Memo., 47 pp. [Available from National Environmental Satellite, Data, and Information Service, Washington, DC 20233; online at ftp://satepsanone. nesdis.noaa.gov/Publications/Tropical/Dvorak_1984.pdf.]

Emanuel, K., 1995: The behavior of a simple hurricane model using a convective scheme based on subcloud-layer entropy equilibrium. J. Atmos. Sci., 52, 3959-3968.

_, 2003: Tropical cyclones. Annu. Rev. Earth Planet. Sci., 31, 75-104.

— climate. Preprints, 26th Conf. on Hurricanes and Tropical Meteorology, Miami, FL, Amer. Meteor. Soc., 10A.2. [Available online at http://ams.confex.com/ams/pdfpapers/75463.pdf.]

Errico, R. M., 1985: Spectra computed from a limited area grid. Mon. Wea. Rev., 113, 1554-1562.

Evans, J. L., and R. J. Allan, 1992: El Niño/Southern Oscillation modification to the structure of the monsoon and tropical cyclone activity in the Australasian region. Int. J. Climatol., 12, 611-623.

Gallus, W. A., 1999: Eta simulations of three extreme precipitation events: Sensitivity to resolution and convective parameterization. Wea. Forecasting, 14, 405-426.

Giorgi, F., and X. Bi, 2000: A study of internal variability of a regional climate model. J. Geophys. Res., 105(D24), 29 503-29 521.

Gray, W. M., 1979: Hurricanes: Their function, structure and likely role in the tropical circulation. Supplement of Meteorology over the Tropical Oceans, D. B. Shaw, Ed., Royal Meteorological Society, 155-218.

- 1998: The formation of tropical cyclones. Meteor. Atmos. Phys., 67, 37-69.

Grell, G., and D. Dévényi, 2002: A generalized approach to parameterizing convection combining ensemble and data assimilation techniques. Geophys. Res. Lett., 29, 1693, doi:10.1029/ 2002GL015311.

Guinn, T. A., and W. H. Schubert, 1993: Hurricane spiral bands. J. Atmos. Sci., 50, 3380-3403.

Halverson, J. B., J. Simpson, G. Heymsfield, H. Pierce, T. Hock, and L. Ritchie, 2006: Warm core structure of Hurricane Erin diagnosed from high altitude dropsondes during CAMEX-4. J. Atmos. Sci., 63, 309-324.

Hawkins, H. F., and S. M. Imbembo, 1976: The structure of a small, intense hurricane-Inez 1966. Mon. Wea. Rev., 104, 418-442.

Holland, G. J., and M. Lander, 1993: The meandering nature of tropical cyclone tracks. J. Atmos. Sci., 50, 1254-1266.

Hong, S. Y., and H. L. Pan, 1998: Convective trigger function for a mass-flux cumulus parameterization scheme. Mon. Wea. Rev., 126, 2599-2620.

— J. Judhia, and S. H. Chen, 2004: A revised approach to ice microphysical processes for the bulk parameterization of clouds and precipitation. Mon. Wea. Rev., 132, 103-120.

Huffman, G. J., and Coauthors, 1997: The Global Precipitation Climatology Project (GPCP) Combined Precipitation Dataset. Bull. Amer. Meteor. Soc., 78, 5-20.

Janjić, Z. I., 1994: The step-mountain eta coordinate model: Further developments of the convection, viscous sublayer, and turbulence closure schemes. Mon. Wea. Rev., 122, $927-$ 945.

_ 2000: Comments on "Development and evaluation of a convection scheme for use in climate models." J. Atmos. Sci., 57, 3686.

Jiang, H., and E. J. Zipser, 2010: Contribution of tropical cyclones to the global precipitation from eight seasons of TRMM data: Regional, seasonal, and interannual variations. J. Climate, 23, 1526-1543.

Jorgensen, D. P., E. J. Zipser, and M. A. Le Mone, 1985: Vertical motions in intense hurricanes. J. Atmos. Sci., 42, 839-856.

Kain, J. S., 2004: The Kain-Fritsch convective parameterization: An update. J. Appl. Meteor., 43, 170-181.

Kanamitsu, M., W. Ebisuzaki, J. Woollen, S.-K. Yang, J. J. Hnilo, M. Fiorino, and G. L. Potter, 2002: NCEP-DOE AMIP-II Reanalysis (R-2). Bull. Amer. Meteor. Soc., 83, 1631-1643.

Kelley, O. A., J. Stout, and J. B. Halverson, 2004: Tall precipitation cells in tropical cyclone eyewalls are associated with tropical cyclone intensification. Geophys. Res. Lett., 31, L24112, doi:10.1029/2004GL021616.

Kidder, S. Q., M. D. Goldberg, R. M. Zehr, M. DeMaria, J. F. W. Purdom, C. S. Velden, N. C. Grody, and S. J. Kusselson, 2000: Satellite analysis of tropical cyclones using the Advanced Microwave Sounding Unit (AMSU). Bull. Amer. Meteor. Soc., 81, 1241-1259.

Knutson, T. R., J. J. Sirutis, S. T. Garner, I. M. Held, and R. E. Tuleya, 2007: Simulation of the recent multidecadal increase of Atlantic hurricane activity using an 18-km-grid regional model. Bull. Amer. Meteor. Soc., 88, 1549-1565.

Kuleshov, Y., L. Qi, R. Fawcett, and D. Jones, 2008: On tropical cyclone activity in the Southern Hemisphere: Trends and the ENSO connection. Geophys. Res. Lett., 35, L14S08, doi:10.1029/ 2007GL032983.

Kuo, H.-C., G. T.-J. Chen, and C.-H. Lin, 2000: Merger of Tropical Cyclones Zeb and Alex. Mon. Wea. Rev., 128, 2967-2975.

Landsea, C. W., 1993: A climatology of intense (or major) Atlantic hurricanes. Mon. Wea. Rev., 121, 1703-1713.

_- G. A. Vecchi, L. Bengtsson, and T. R. Knutson, 2010: Impact of duration thresholds on Atlantic tropical cyclone counts. J. Climate, 23, 2508-2519.

LaRow, T. E., Y. K. Lim, D. W. Shin, E. P. Chassignet, and S. Cocke, 2008: Atlantic basin seasonal hurricane simulations. J. Climate, 21, 3191-3206.

Lefevre, J., P. Marchesiello, N. C. Jourdain, C. E. Menkes, and A. Leroy, 2010: Weather regimes and orographic circulation around New Caledonia. Mar. Pollut. Bull., 61, 413-431. 
Lucas-Picher, P., D. Caya, R. de Elìa, and R. Laprise, 2008: Investigation of regional climate models' internal variability with a ten-member ensemble of 10-year simulations over a large domain. Climate Dyn., 31, 927-940.

Marks, F. D., 1981: Evolution of the structure of precipitating convection in Hurricane Allen. Preprints, 20th Conf. on Radar Meteorology, Boston, MA, Amer. Meteor. Soc., 720-725.

Matthews, A. J., B. J. Hoskins, J. M. Slingo, and M. Blackburn, 1996: Development of convection along the SPCZ within a MaddenJulian oscillation. Quart. J. Roy. Meteor. Soc., 122, 669-688.

Mlawer, E. J., S. J. Taubman, P. D. Brown, M. J. Iacono, and S. A. Clough, 1997: Radiative transfer for inhomogeneous atmospheres: RRTM, a validated correlated-k model for the longwave. J. Geophys. Res., 102, 16 663-16 682.

Moon, I. J., I. Ginis, and T. Hara, 2004: Effect of surface waves on air-sea momentum exchange. Part II: Behavior of drag coefficient under tropical cyclones. J. Atmos. Sci., 61, 2334-2348.

Nguyen, K. C., and K. J. E. Walsh, 2001: Interannual, decadal, and transient greenhouse simulation of tropical cyclone-like vortices in a regional climate model of the South Pacific. J. Climate, 14, 3043-3054.

Nicholls, N., 1984: The Southern Oscillation, sea surface temperature, and interannual fluctuations in Australian tropical cyclone activity. Int. J. Climatol., 4, 661-670.

Noh, Y., W. G. Cheon, S. Y. Hong, and S. Raasch, 2003: Improvement of the K-profile model for the planetary boundary layer based on large eddy simulation data. Bound.-Layer Meteor., 107, 401-427.

Oouchi, K., J. Yoshimura, H. Yoshimura, R. Mizuta, S. Kusunoki, and A. Noda, 2006: Tropical cyclone climatology in a globalwarming climate as simulated in a $20 \mathrm{~km}$ - mesh global atmospheric model: Frequency and wind intensity analyses. J. Meteor. Soc. Japan, 84, 259-276.

Ooyama, K. V., 1982: Conceptual evolution of the theory and modeling of the tropical cyclone. J. Meteor. Soc. Japan, 60, 369-379.

Orlanski, I., 1975: A rational subdivision of scales for atmospheric processes. Bull. Amer. Meteor. Soc., 56, 527-530.

Powell, M. D., P. J. Vickery, and T. A. Reinhold, 2003: Reduced drag coefficient for high wind speeds in tropical cyclones. Nature, 422, 279-283.

Ramsay, H. A., L. M. Leslie, P. J. Lamb, M. B. Richman, and M. Leplastrier, 2008: Interannual variability of tropical cyclones in the Australian region: Role of large-scale environment. J. Climate, 21, 1083-1103.

Royer, J.-F., F. Chauvin, B. Timbal, P. Araspin, and D. Grimal, 1998: A GCM study of the impact of greenhouse gas increase on the frequency of occurrence of tropical cyclones. Climatic Change, 38, 307-343.

Shapiro, S. S., and M. B. Wilk, 1965: An analysis of variance test for normality (complete samples). Biometrika, 52 (3-4), 591-611.
Simpson, J., E. Ritchie, G. J. Holland, J. Halverson, and S. Stewart, 1997: Mesoscale interactions in tropical cyclone genesis. Mon. Wea. Rev., 125, 2643-2661.

Skamarock, W. C., 2004: Evaluating mesoscale NWP models using kinetic energy spectra. Mon. Wea. Rev., 132, 3019-3032.

—, and J. B. Klemp, 2008: A time-split nonhydrostatic atmospheric model for weather research and forecasting applications. J. Comput. Phys., 227, 3465-3485.

— - - J. Dudhia, D. O. Gill, D. M. Barker, W. Wang, and J. G. Powers, 2005: A description of the Advanced Research WRF version 2. NCAR Tech. Notes-468+ STR, 100 pp. [Available online at http://www.mmm.ucar.edu/wrf/users/docs/arw_v2. pdf.]

Sugi, M., A. Noda, and N. Sato, 2002: Influence of the global warming on tropical cyclone climatology: An experiment with the JMA global model. J. Meteor. Soc. Japan, 80, 249-272.

Vincent, E. M., M. Lengaigne, C. E. Menkes, N. C. Jourdain, P. Marchesiello, and G. Madec, 2009: Interannual variability of the South Pacific convergence zone and implications for tropical cyclone genesis. Climate Dyn., doi:10.1007/s00382-0090716-3, in press.

Vitart, F., J. L. Anderson, and W. F. Stern, 1997: Simulation of interannual variability of tropical storm frequency in an ensemble of GCM integrations. J. Climate, 10, 745-760.

von Storch, H., and F. W. Zwiers, 2001: Statistical Analysis in Climate Research. Cambridge University Press, 484 pp.

- H. Langenberg, and F. Feser, 2000: A spectral nudging technique for dynamical downscaling purposes. Mon. Wea. Rev., 128, 3664-3673.

Walsh, K. J. E., K.-C. Nguyen, and J. L. McGregor, 2004: Fineresolution regional climate model simulations of the impact of climate change on tropical cyclones near Australia. Climate Dyn., 22, 47-56.

—, M. Fiorino, C. W. Landsea, and K. L. McInnes, 2007: Objectively determined resolution-dependent threshold criteria for the detection of tropical cyclones in climate models and reanalyses. J. Climate, 20, 2307-2314.

Watterson, I. G., J. L. Evans, and B. F. Ryan, 1995: Seasonal and interannual variability of tropical cyclogenesis: Diagnostics from large scale fields. J. Climate, 8, 3052-3065.

Wu, G., and N. C. Lau, 1992: A GCM simulation of the relationship between tropical-storm formation and ENSO. Mon. Wea. Rev., 120, 958-977.

Yoshimura, J., M. Sugi, and A. Noda, 2006: Influence of greenhouse warming on tropical cyclone frequency. J. Meteor. Soc. Japan, 84, 405-428.

Zhao, M., I. M. Held, S. J. Lin, and G. A. Vecchi, 2009: Simulations of global hurricane climatology, interannual variability, and response to global warming using a $50-\mathrm{km}$ resolution GCM. J. Climate, 22, 6653-6678. 